\title{
EPR of Compound I: An Illustrated Revision of the Theoretical Model
}

\section{Maruan Bracci $^{1}$ (D) . Sabine Van Doorslaer ${ }^{2}$ (D) - Inés García-Rubio ${ }^{1,3}$ (D)}

Received: 16 July 2020 / Revised: 22 September 2020 / Accepted: 27 September 2020 /

Published online: 6 November 2020

(c) The Author(s) 2020

\begin{abstract}
Compound I has been postulated to be the reactive species in many heme catalysts, which performs different chemistry and shows different properties in different enzymes. The aim of this review is to present a comprehensive model which has been successfully used to interpret the EPR spectra of various Compound I species. The theoretical approach established by seminal articles will be revisited and its ability to explain experimental results will be illustrated by simulating selected spectra from the literature. Compound I stores two oxidizing equivalents, one in the paramagnetic iron(IV)-oxo moiety, and another one as a free radical on the porphyrin ligand or an amino acid in the protein. To describe the interactions of the two paramagnetic species with each other and with their local environment, the spin Hamiltonian of the system is built step by step. The Fe(IV) center is described using a two-hole model. The effect of the crystal-field and spin-orbit coupling on the energy levels is calculated with this simple approach, which allows to obtain spin Hamiltonian parameters like zero-field splitting and effective $g$-values for the iron. The magnetic interaction between the Fe(IV) center and the free radical is considered and allowed to vary in sign (ferromagnetic to antiferromagnetic) and magnitude to interpret the EPR of Compound I species in different systems. Since orbital overlap is crucial for exchange interaction, special emphasis is made in obtaining the orientation of Fe semi-occupied orbitals by extending the counter-rotation concept, which relates the directions of magnetic, electronic, and molecular axes.
\end{abstract}

\section{Abbreviations}

HRP Horseradish peroxidase

P450 Cytochrome P450

Inés García-Rubio

inesgr@unizar.es

1 Department of Condensed Matter Physics, Faculty of Sciences, University of Zaragoza, Calle Pedro Cerbuna, 50009 Zaragoza, Spain

2 BIMEF Laboratory, Department of Chemistry, University of Antwerp, 2610 Antwerp, Belgium

3 Centro Universitario de la Defensa, Ctra de Huesca s/n, 50090 Zaragoza, Spain 


$\begin{array}{ll}\text { CPO } & \text { Chloroperoxidase } \\ \text { Cpd-I } & \text { Compound I } \\ \text { HRP-I } & \text { HRP Compound I } \\ \text { P450-I } & \text { P450 Compound I } \\ \text { CPO-I } & \text { CPO Compound I } \\ \text { MCL } & \text { Catalase from Micrococcus luteus (also called Micrococcus } \\ & \text { lysodeikticus) } \\ \text { PMC } & \text { Catalase from Proteus mirabilis } \\ \text { BLC } & \text { Catalase from bovine liver } \\ \text { MCL-I } & \text { MCL Compound I } \\ \text { PMC-I } & \text { PMC Compound I } \\ \text { BLC-I } & \text { BLC Compound I } \\ \text { RFQ } & \text { Rapid freeze quench } \\ \text { CYP119 } & \text { P450 from the thermophilic organisms Sulfolobus acidocaldarius } \\ \text { P450cam } & \text { Cytochrome P450 camphor } \\ \text { EPR } & \text { Electron paramagnetic resonance } \\ \text { ENDOR } & \text { Electron nuclear double resonance } \\ \text { ESEEM } & \text { Electron spin echo envelope modulation } \\ V_{c} & \text { Crystal field } \\ \text { FWHM } & \text { Full width at half maximum } \\ \text { SO } & \text { Spin-orbit } \\ \text { ZFS } & \text { Zero-field splitting } \\ \text { QM/MM } & \text { Quantum mechanics/molecular mechanics } \\ \text { AA } & \text { Amino acid }\end{array}$

\section{Introduction}

Due to the growing need to find eco-friendly ways of circumventing common drawbacks of industrial catalysts, the strategy of learning from nature, either by directly making use of biocatalysts or by mimicking their working, seems to be interesting, since enzymes operate in aqueous solutions, under physiological conditions, and are assumed to be highly sustainable, biodegradable, and non-toxic, while producing pure products with high specificity. Particularly rich and interesting is the chemistry performed by heme enzymes. The iron center in the heme cofactor occurs in different oxidation states with varying distal and proximal ligations whereby the properties of the metal ion are modulated by the protein matrix. This is due to the nature of the porphyrin ligand, which provides a crystal field that sets the ion close to a high-spin-low-spin transition; hence, the electronic behavior of the iron porphyrin is highly sensitive to the local protein environment. In heme proteins, it is quite usual to find crystal-field potentials close in energy to electronic repulsion and spin-orbit coupling energy terms. In this state, the protein environment controls the electronic structure. Especially important are the nature and orientation of the iron axial ligands.

The varying electronic structure of the heme iron translates directly into a multitude of functions performed by heme proteins. In particular, in heme enzymes, the 


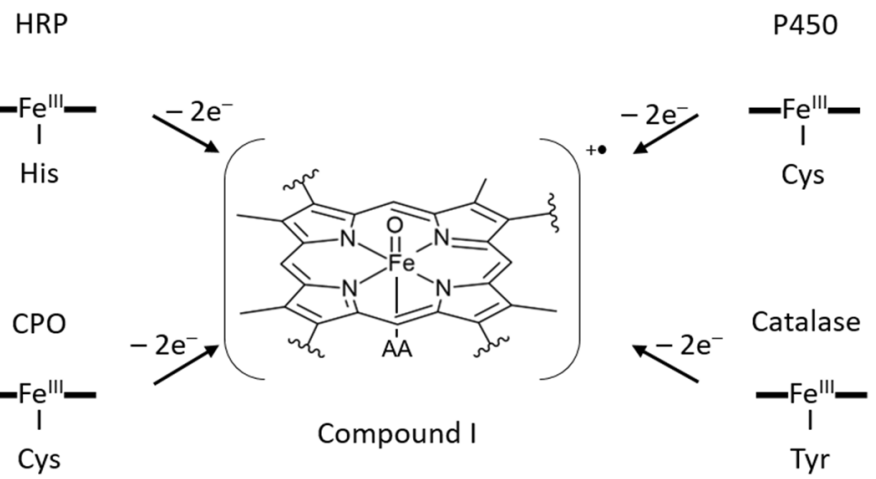

Fig. 1 Different proteins react with peroxide to generate the same active intermediate, Compound I. The cation $\pi$-radical is stored in the ligands of the iron; it can migrate to an amino acid of the protein (often called Compound $\mathrm{I}^{*}$ ), but these cases are not subject to the review. In the four edges of the image, - Fe represents the porphyrin ring coordinated with the iron, the proximal ligand is indicated, and the sixth ligand (usually a water molecule) is omitted. In the center figure, AA indicates the proximal amino acid ligand, which changes depending on the protein. Compound I reacts quickly to continue the catalytic cycle

same heme active species is tuned towards different reactions by variations in the protein matrix. In many heme enzymes, such as heme peroxidases, the key intermediate state has been postulated to be the so-called Compound I (Cpd-I), which stores two oxidizing equivalents, one in the form of an iron(IV)-oxo species and another one in a cationic free radical residing either in a conjugate $\pi$-orbital of the porphyrin or in an axial ligand (see Fig. 1). In some cases, this last equivalent migrates from the heme site to a reactive tryptophan or tyrosine somewhere in the protein matrix. The latter state is often called Compound I* or compound ES [1, 2].

Electron paramagnetic resonance (EPR) has played a key role in revealing the nature of Compound I since both oxidizing equivalents of this intermediate form paramagnetic species. In general, EPR is a privileged technique for studying hemecontaining proteins, since, despite the overall complexity of these macromolecules, it allows focusing specifically on the active site, where the unpaired electrons reside. In particular, for Cpd-I, it could reveal information on each of the two paramagnetic sites as well as the interaction between them, when the intermediate is efficiently trapped. Combination with the study of hyperfine interactions between magnetic nuclei of the environment using ENDOR (electron-nuclear double resonance) or ESEEM (electron spin echo envelope modulation) techniques can map the electron density, identifying the location of the semi-occupied orbitals, which helps understanding the mechanistic processes behind catalysis.

EPR spectra of the different Cpd-I (and Cpd-I*) reported to date reflect the variety of behavior mentioned above, which, in turn, makes the technique extremely informative to study this system. On the other hand, straight-forward interpretations are difficult to extract. Instead, the use of an electronic model validated by experimental data is absolutely necessary to explain the experimentally observable interaction between the unpaired electrons of the iron ion and the radical. The parameterization of physical and quantum-mechanical phenomena is fundamental to 


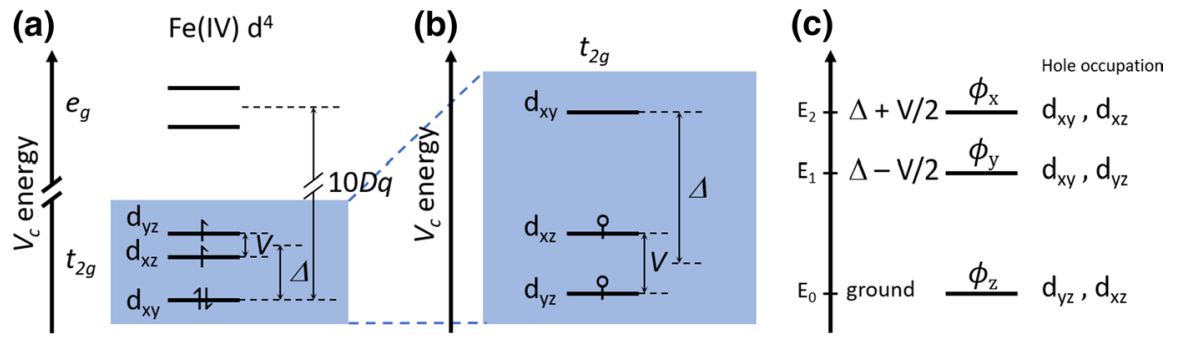

Fig. 2 a Crystal-field energy splitting and orbital occupation in a $\mathrm{Fe}(\mathrm{IV})\left(d^{4}\right)$ ion in $C_{2 v}$ symmetry. The definitions of parameters $\Delta$ and $V$ are indicated in figure. $10 D q$ is the octahedral crystal-field parameter, much bigger than $V$ and $\Delta$ because of the large energy gap between $t_{2 g}$ and $e_{g}$ orbitals. As a consequence, the $e_{g}$ orbitals are not populated and the iron is in a low-spin configuration. b Two-hole model for the $\mathrm{Fe}$ (IV) low-spin center, focusing only in the $t_{2 g}$ orbitals. c Crystal field energy levels for the two-hole states of $\mathrm{Fe}(\mathrm{IV})$ low-spin configuration

understand the extent to which they contribute to the recorded experimental EPR signal. The combination of a per se rich behavior of the iron with the possibilities for the radical site and the interaction between them gives rise to countless possibilities. The role of the exchange interaction, for which the spatial location of the semioccupied orbitals is extremely important, is of particular interest, because it occurs in all of the examined proteins, giving rise to a varying degree of ferromagnetic to antiferromagnetic interaction.

In this work, we review the construction of the electronic model for Cpd-I, summarizing seminal developments of several essential articles in a compilation of the general model for Cpd-I. The ability of the model to describe the experiments will be illustrated with data from the literature for several cases of emblematic heme enzymes with different coordination schemes for which the Cpd-I could be brought to scrutiny. Emphasis is put on EPR results, although the model is also validated by experimental data from other techniques such as Mössbauer spectroscopy, UV-visible optical absorption, and crystallographic data.

\section{Theoretical Model of the Paramagnetic Centers}

\subsection{Fe(IV)-Oxo Complex in $C_{2 v}$ Symmetry}

The magnetic properties of the iron can be described using a crystal-field formalism [3]. The iron Fe(IV) has a low-spin (3d) $)^{4}$ configuration. This means that the d-orbitals are split into two groups $\left(t_{2 g}\right.$ and $e_{g}$ orbitals) according to their energy as a result of a coordination environment of roughly octahedral symmetry and that the $e_{g}$ orbitals are far above in energy, so that all four electrons are accommodated in the low-lying $t_{2 g}$ orbitals. The energy difference between the two sets of orbitals is identified as $10 D q$. Axial and rhombic lower-symmetry contributions to the crystal field $\left(V_{c}\right)$ further split the energy of the orbitals which are occupied by the electrons in a way to have the maximum spin multiplicity $(S=1)$, as shown in Fig. 2a. This configuration reflects the 
(a)

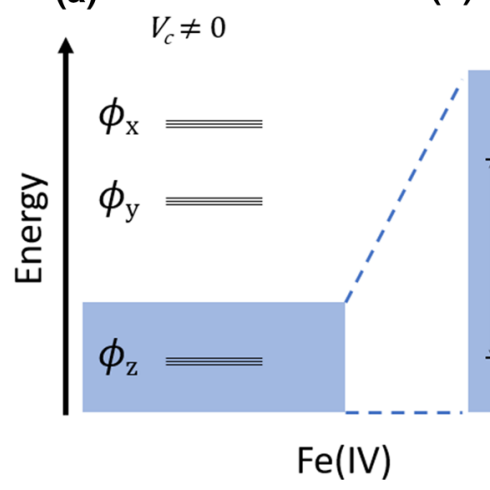

(b)

$$
V_{c}, D \neq 0 \quad V_{c}, D, B \neq 0
$$

(c)

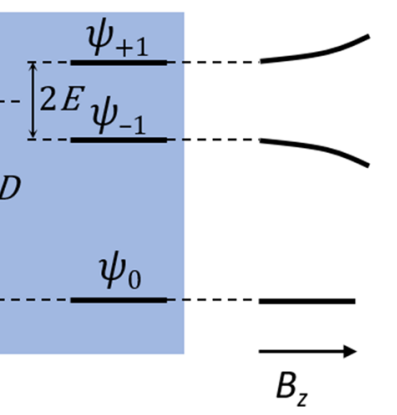

Fig. 3 Two-electron states of Fe(IV). a Spin triplets in a $V_{c}$ potential. b Energy splitting of the ground state due to spin-orbit coupling. c Effect of a magnetic field along $z$-axis $\left(\mathrm{B}_{\mathrm{z}}\right)$ on the energy of the ground-state levels of $\mathrm{Fe}(\mathrm{IV})$

fact that the electron repulsion (exchange energy) is larger than the energy difference between the $t_{2 g}$ orbitals, quantified by the axial $(\Delta)$ and rhombic $(V)$ crystal-field terms. The treatment of the present four-electron problem can be largely simplified by considering it as a two parallel-hole problem in a filled $\left(t_{2 g}\right)^{6}$ subshell. We will adopt this hole treatment from here on, for which the one-hole orbital energy levels are depicted in Fig. 2b. Taking into account that the unpaired electrons (or holes) have to be parallel, there are three different possible ways of filling the $t_{2 g}$ orbitals, corresponding to the three states depicted in Fig. 2c.

Since the total spin is known to be $S=1$, the spin part of the two-hole wave function can be either:

$$
\chi_{1}^{1}=|\uparrow\rangle|\uparrow\rangle ; \chi_{-1}^{1}=|\downarrow\rangle|\downarrow\rangle ; \chi_{0}^{1}=\frac{1}{\sqrt{2}}(|\downarrow\rangle|\uparrow\rangle+|\uparrow\rangle|\downarrow\rangle),
$$

and therefore, the energy levels in Fig. 2c are spin triplets (Fig. 3a). Note that the spin-wave functions are symmetric against the permutation of the particles. This means that, since the total wave function must be antisymmetric, the orbital part of the wave function must be antisymmetric with respect to permutation. Consequently, there are three possibilities for the orbital wave function:

$$
\begin{aligned}
& \phi_{x}=\frac{1}{\sqrt{2}} i\left(\left|d_{x y}\right\rangle\left|d_{x z}\right\rangle-\left|d_{x z}\right\rangle\left|d_{x y}\right\rangle\right) \\
& \phi_{y}=\frac{1}{\sqrt{2}}\left(\left|d_{x y}\right\rangle\left|d_{y z}\right\rangle-\left|d_{y z}\right\rangle\left|d_{x y}\right\rangle\right) \\
& \phi_{z}=\frac{1}{\sqrt{2}} i\left(\left|d_{y z}\right\rangle\left|d_{x z}\right\rangle-\left|d_{x z}\right\rangle\left|d_{y z}\right\rangle\right),
\end{aligned}
$$


where the first part of the functions refers to the first electron (hole) and the second part to the second electron (hole), and $\left|d_{i j}\right\rangle$ are the $t_{2 g}$ orbitals, which are defined with respect to the eigenstates of $l_{z}$ as follows, assuming only the angular dependent part of the wave function ( $\theta$ and $\varphi$ represent, respectively, the colatitude and azimuth spherical coordinates in relation to the molecular axes, as detailed later in the text):

$$
\begin{aligned}
& \left|d_{y z}\right\rangle=\frac{i}{\sqrt{2}}\{|1\rangle+|-1\rangle\}=\sqrt{\frac{15}{16 \pi}} \sin 2 \theta \sin \varphi \\
& \left|d_{x z}\right\rangle=-\frac{1}{\sqrt{2}}\{|1\rangle-|-1\rangle\}=\sqrt{\frac{15}{16 \pi}} \sin 2 \theta \cos \varphi \\
& \left|d_{x y}\right\rangle=\frac{-i}{\sqrt{2}}\{|2\rangle-|-2\rangle\}=\sqrt{\frac{15}{16 \pi}} \sin ^{2} \theta \sin 2 \varphi,
\end{aligned}
$$

where $|1\rangle=Y_{1}^{2},|-1\rangle=Y_{-1}^{2},|2\rangle=Y_{2}^{2},|-2\rangle=Y_{-2}^{2}$.

From Fig. 2c, one can see that the crystalline field term will favor the occupancy of orbitals $d_{y z}$ and $d_{x z}$, that is, the state $\phi_{z}$. The expression of the crystal-field energy term $V_{c}$ in the $\left\{\phi_{x}, \phi_{y}, \phi_{z}\right\}$ basis set is:

$$
V_{c}=\left(\begin{array}{ccc}
\Delta+\frac{V}{2} & 0 & 0 \\
0 & \Delta-\frac{V}{2} & 0 \\
0 & 0 & 0
\end{array}\right)
$$

Combining the three possible orbital functions $\left\{\phi_{x}, \phi_{y}, \phi_{z}\right\}$ with the three possible spin parts $\left\{\chi_{1}^{1}, \chi_{0}^{1}, \chi_{-1}^{1}\right\}$, a nine-element basis set can be built to describe the effects of the spin-orbit (SO) and electron Zeeman (EZ) terms of the Hamiltonian:

$$
\widehat{\mathcal{H}}=\sum_{k=1}^{2} \hat{V}_{c, k}+\sum_{k=1}^{2}(-\lambda) \widehat{\vec{\ell}}_{k} \widehat{\vec{s}}_{k}+\mu_{B} \vec{B} \sum_{k=1}^{2}\left(\widehat{\vec{\ell}}_{k}+g_{e} \widehat{\vec{s}}_{k}\right)
$$

where the first term is the crystal field, the second one is the spin-orbit coupling, and the third one is the electron Zeeman term. Note that $\lambda$, the one-electron spin-orbit coupling constant, bears a minus sign originating from working with the hole model, $\mu_{\mathrm{B}}$ is the Bohr magneton, and $\widehat{\vec{\ell}}$ and $\widehat{\overrightarrow{\mathrm{s}}}$ are the one-electron orbital and spin angular momentum operators, respectively. $\overrightarrow{\mathrm{B}}$ is the applied magnetic field.

As opposed to the crystal-field energy, the matrix expression of the crystal field plus the spin-orbit coupling terms of the Hamiltonian in this nine-element basis set is not diagonal ${ }^{1}$ :

\footnotetext{
1 Please note that, in this matrix, the angular part is not expressed in terms of angular momentum eigenstates $Y_{i}^{2}$ but in the basis set $\left\{\phi_{x}, \phi_{y}, \phi_{z}\right\}$ based on $d$ orbitals as defined in Eqs. 2 and 3. Therefore, $\hat{\ell}_{z}$ is not diagonal in this representation and, consequently, the only terms in the diagonal are the crystal field terms.
} 


$$
\begin{aligned}
& \begin{array}{llllllllll}
\phi_{x} \chi_{1}^{1} & \phi_{x} \chi_{0}^{1} & \phi_{x} \chi_{-1}^{1} & \phi_{y} \chi_{1}^{1} & \phi_{y} \chi_{0}^{1} & \phi_{y} \chi_{-1}^{1} & \phi_{z} \chi_{1}^{1} & \phi_{z} \chi_{0}^{1} & \phi_{z} \chi_{-1}^{1}
\end{array} \\
& \begin{array}{l}
\phi_{x} \chi_{1}^{1} \\
\phi_{x} \chi_{0}^{1} \\
\phi_{x} \chi_{-1}^{1} \\
\phi_{y} \chi_{1}^{1} \\
\phi_{y} \chi_{0}^{1} \\
\end{array}
\end{aligned}
$$

As a consequence of the spin-orbit interaction, the ground-state triplet is split in three separate levels. The energies can be calculated via diagonalization of the Hamiltonian above, which allows obtaining the energies of the three lowest levels $\left\{\psi_{1}, \psi_{0}, \psi_{-1}\right\}$ that are the ones relevant to the magnetic properties (see Fig. 3b). Additionally, mixing of the levels is brought by the SO interaction. The non-zero elements of the matrix connect the levels that get mixed by the interaction. It is illustrative to take a closer look at the effect of the SO coupling on the unperturbed ground triplet $\left\{\phi_{z} \chi_{1}^{1}, \phi_{z} \chi_{0}^{1}, \phi_{z} \chi_{-1}^{1}\right\}$ using degenerate perturbation theory to second order:

$$
\begin{aligned}
& \widehat{\mathcal{H}}_{S O}=\frac{\lambda^{2}}{4}\left(\begin{array}{ccc}
\phi_{z} \chi_{1}^{1} & \phi_{z} \chi_{0}^{1} & \phi_{z} \chi_{-1}^{1} \\
\frac{1}{2}\left(\frac{1}{-\left(\Delta-\frac{\mathrm{V}}{2}\right)}+\frac{1}{-\left(\Delta+\frac{\mathrm{V}}{2}\right)}\right) & 0 & \frac{1}{2}\left(\frac{1}{-\left(\Delta-\frac{\mathrm{V}}{2}\right)}-\frac{1}{-\left(\Delta+\frac{\mathrm{V}}{2}\right)}\right) \\
0 & \left(\frac{1}{-\left(\Delta-\frac{\mathrm{V}}{2}\right)}+\frac{1}{-\left(\Delta+\frac{\mathrm{V}}{2}\right)}\right) & 0 \\
\frac{1}{2}\left(\frac{1}{-\left(\Delta-\frac{\mathrm{v}}{2}\right)}-\frac{1}{-\left(\Delta+\frac{\mathrm{V}}{2}\right)}\right) & 0 & \frac{1}{2}\left(\frac{1}{-\left(\Delta-\frac{\mathrm{V}}{2}\right)}+\frac{1}{-\left(\Delta+\frac{\mathrm{V}}{2}\right)}\right)
\end{array}\right)= \\
& =\lambda^{2}\left(\begin{array}{ccc}
\left(\frac{-\Delta}{4 \Delta^{2}-V^{2}}\right) & 0 & \frac{1}{2}\left(\frac{-V}{4 \Delta^{2}-V^{2}}\right) \\
0 & \left(\frac{-2 \Delta}{4 \Delta^{2}-V^{2}}\right) & 0 \\
\frac{1}{2}\left(\frac{-V}{4 \Delta^{2}-V^{2}}\right) & 0 & \left(\frac{-\Delta}{4 \Delta^{2}-V^{2}}\right)
\end{array}\right)
\end{aligned}
$$

where the matrix has been calculated using the perturbed states to first order:

$$
\psi_{+1}=\phi_{\mathrm{z}} \chi_{1}+\left(\frac{\lambda}{2 \sqrt{2}}\right)\left(\frac{\phi_{\mathrm{y}} \chi_{0}}{0-\left(\Delta-\frac{\mathrm{v}}{2}\right)}+\frac{\phi_{\mathrm{x}} \chi_{0}}{0-\left(\Delta+\frac{\mathrm{v}}{2}\right)}\right)
$$




$$
\begin{gathered}
\psi_{0}=\phi_{\mathrm{z}} \chi_{0}+\left(\frac{\lambda}{2 \sqrt{2}}\right)\left(\frac{\phi_{\mathrm{y}}\left(\chi_{-1}+\chi_{+1}\right)}{0-\left(\Delta-\frac{\mathrm{V}}{2}\right)}+\frac{\phi_{\mathrm{x}}\left(\chi_{-1}-\chi_{+1}\right)}{0-\left(\Delta+\frac{\mathrm{v}}{2}\right)}\right) \\
\psi_{-1}=\phi_{\mathrm{z}} \chi_{-1}+\left(\frac{\lambda}{2 \sqrt{2}}\right)\left(\frac{\phi_{\mathrm{y}} \chi_{0}}{0-\left(\Delta-\frac{\mathrm{V}}{2}\right)}-\frac{\phi_{\mathrm{x}} \chi_{0}}{0-\left(\Delta+\frac{\mathrm{v}}{2}\right)}\right) .
\end{gathered}
$$

In the spin Hamiltonian formalism, an operator only dependent on the spin variables is used to calculate the energies of these three levels, which are the relevant ones for EPR, making the equivalence between the real levels and the three states of an imaginary or effective spin system. In this case, since the ground level is a triplet, we use an effective spin $S_{\text {eff }}^{\mathrm{Fe}}=1$. The zero-field splitting (ZFS) term of the spin Hamiltonian is normally written as follows when expressed in the reference frame where the ZFS tensor is diagonal and can be defined by the parameters $D$ and $E$ :

$$
\begin{aligned}
\hat{H}_{\mathrm{ZFS}}^{S}= & \widehat{S_{\mathrm{eff}}^{\mathrm{Fe}}} \mathbf{D} \widehat{\widehat{\mathrm{Fe}}}=D\left(\left(\widehat{S_{\mathrm{eff}}^{\mathrm{Fe}}}\right)^{2}-\frac{1}{3} S_{\mathrm{eff}}^{\mathrm{Fe}}\left(S_{\mathrm{eff}}^{\mathrm{Fe}}+1\right) \hat{1}\right) \\
& +E\left(\left(\widehat{S_{\mathrm{eff}_{x}}^{\mathrm{Fe}}}\right)^{2}-\left(\widehat{S_{\mathrm{eff}_{y}}^{\mathrm{Fe}}}\right)^{2}\right)=\left(\begin{array}{ccc}
D / 3 & 0 & E \\
0 & -2 D / 3 & 0 \\
E & 0 & D / 3
\end{array}\right)
\end{aligned}
$$

Since this expression of the ZFS in the spin Hamiltonian is traceless and the SO perturbation is not, if an offset of $\left(\frac{4 \Delta / 3}{4 \Delta^{2}-\mathrm{V}^{2}}\right)$ is added to each of the diagonal components of Eq. (7), the first-order expressions of the ZFS parameters $D$ and $E$ are obtained:

$$
D=\frac{\lambda^{2} \Delta}{4 \Delta^{2}-\mathrm{V}^{2}} ; E=\frac{\lambda^{2}}{2}\left(\frac{-\mathrm{V}}{4 \Delta^{2}-\mathrm{V}^{2}}\right) .
$$

Although mixing of the orbital parts is limited if the crystal-field parameters are large with respect to the spin-orbit coupling constant, the spin part of the eigenstates is heavily admixed in the absence of an applied magnetic field. If we note with $a, b$, and $c$ the coefficients of the ZFS eigenstates obtained by exact diagonalization of Eq. (6):

$$
\begin{gathered}
\psi_{0}=a_{0} \phi_{z} \chi_{0}^{1}-b_{0} \phi_{y} \frac{1}{\sqrt{2}}\left(\chi_{1}^{1}+\chi_{-1}^{1}\right)+c_{0} \phi_{x} \frac{1}{\sqrt{2}}\left(\chi_{1}^{1}-\chi_{-1}^{1}\right) \\
\psi_{-1}=a_{-} \phi_{z} \frac{1}{\sqrt{2}}\left(\chi_{1}^{1}+\chi_{-1}^{1}\right)-b_{-} \phi_{y} \chi_{0}^{1}+0 \phi_{x} \chi_{0}^{1} \\
\psi_{+1}=a_{+} \phi_{z} \frac{1}{\sqrt{2}}\left(\chi_{1}^{1}-\chi_{-1}^{1}\right)+0 \phi_{y} \chi_{0}^{1}-c_{+} \phi_{x} \chi_{0}^{1} .
\end{gathered}
$$


Upon application of an external magnetic field, the Hamiltonian must also contain the electron Zeeman contribution. In the spin Hamiltonian, all the orbital interactions are parameterized by the tensor $g$ :

$$
\widehat{H}_{E Z}^{S}=\mu_{B} \vec{B} \boldsymbol{g} \widehat{\widehat{S_{\text {eff }}^{F e}}}
$$

To obtain an expression for the $g$-tensor for our system, we have to consider the operator of the Zeeman interaction, that involves the orbital and spin angular momenta, see Eq. (5). For the magnitude of transitions observed at EPR, especially at X-band $\left(\sim 0.3 \mathrm{~cm}^{-1}\right)$, the effect of the magnetic field on the energies of the levels is very small compared to $\Delta$ (over $100 \mathrm{~cm}^{-1}$ ) and $V$, one can calculate the effect of the electron Zeeman term as a first-order perturbation in the ground triplet $\left\{\psi_{0}, \psi_{-1}, \psi_{1}\right\}$. The matrix representation of the angular momentum operators restricted to the triplet is obtained by calculating the $\left\langle\psi_{i}\left|\hat{l}_{k}\right| \psi_{j}\right\rangle$ and $\left\langle\psi_{i}\left|\widehat{s}_{k}\right| \psi_{j}\right\rangle$ terms. Then, a unitary transformation $\mathrm{A}^{-1} \widehat{\mathrm{O}} \mathrm{A}$ has to be applied to express the operators $(\hat{\mathrm{O}})$ in the $\left\{\chi_{1}^{1}, \chi_{0}^{1}, \chi_{-1}^{1}\right\}$ basis set of a $S_{\text {eff }}=1$. The columns of $\mathrm{A}^{-1}$ contain the coordinates of the ZFS eigenstates $\left\{\psi_{1}, \psi_{0}, \psi_{-1}\right\}$ in the $\left\{\chi_{1}^{1}, \chi_{0}^{1}, \chi_{-1}^{1}\right\}$ basis set:

$$
\mathrm{A}^{-1}=\left(\begin{array}{ccc}
0 & 1 / \sqrt{2} & 1 / \sqrt{2} \\
1 & 0 & 0 \\
0 & 1 / \sqrt{2} & -1 / \sqrt{2}
\end{array}\right) .
$$

The matrix representation of the operators in the electron Zeeman term of the spin Hamiltonian is obtained:

$$
\begin{aligned}
& \left\langle\psi_{\mathrm{i}}\left|\hat{L}_{x}+g_{e} \hat{S}_{x}\right| \psi_{\mathrm{j}}\right\rangle=\left(a_{0} b_{-}+a_{-} b_{0}+g_{e}\left(a_{0} a_{-}+b_{0} b_{-}\right)\right)\left(\begin{array}{ccc}
0 & \frac{\sqrt{2}}{2} & 0 \\
\frac{\sqrt{2}}{2} & 0 & \frac{\sqrt{2}}{2} \\
0 & \frac{\sqrt{2}}{2} & 0
\end{array}\right) \\
& =\left(a_{0} b_{-}+a_{-} b_{0}+g_{e}\left(a_{0} a_{-}+b_{0} b_{-}\right)\right) \hat{S}_{\mathrm{eff}_{x}}^{\mathrm{Fe}} \\
& \left\langle\psi_{\mathrm{i}}\left|\hat{L}_{y}+g_{e} \hat{S}_{y}\right| \psi_{\mathrm{j}}\right\rangle=\left(a_{0} c_{+}+a_{+} c_{0}+g_{e}\left(a_{0} a_{+}+c_{0} c_{+}\right)\right)\left(\begin{array}{ccc}
0 & -\frac{\sqrt{2}}{2} i & 0 \\
\frac{\sqrt{2}}{2} i & 0 & -\frac{\sqrt{2}}{2} i \\
0 & \frac{\sqrt{2}}{2} i & 0
\end{array}\right) \\
& =\left(a_{0} c_{+}+a_{+} c_{0}+g_{e}\left(a_{0} a_{+}+c_{0} c_{+}\right)\right) \hat{S}_{\mathrm{eff}_{y}}^{\mathrm{Fe}}
\end{aligned}
$$

$$
\left\langle\psi_{\mathrm{i}}\left|\hat{L}_{z}+g_{e} \hat{S}_{z}\right| \psi_{\mathrm{j}}\right\rangle=\left(-c_{+} b_{-}+g_{e}\left(a_{-} a_{+}\right)\right)\left(\begin{array}{ccc}
1 & 0 & 0 \\
0 & 0 & 0 \\
0 & 0 & -1
\end{array}\right)=\left(-c_{+} b_{-}+g_{e}\left(a_{-} a_{+}\right)\right) \hat{S}_{\mathrm{eff}}^{\mathrm{Fe}}
$$




$$
\begin{aligned}
& \left\langle\psi_{\mathrm{i}}\left|\hat{\vec{L}}+g_{e} \hat{\vec{S}}\right| \psi_{\mathrm{j}}\right\rangle \\
& =\left(\begin{array}{ccc}
a_{0} b_{-}+a_{-} b_{0}+g_{e}\left(a_{0} a_{-}+b_{0} b_{-}\right) & 0 & 0 \\
0 & a_{0} c_{+}+a_{+} c_{0}+g_{e}\left(a_{0} a_{+}+c_{0} c_{+}\right) & 0 \\
0 & 0 & -c_{+} b_{-}+g_{e}\left(a_{-} a_{+}\right)
\end{array}\right) \overrightarrow{\vec{S}}_{\mathrm{eff}}^{\mathrm{Fe}}
\end{aligned}
$$

And therefore, the $\boldsymbol{g}$-tensor is:

$$
\boldsymbol{g}=\left(\begin{array}{ccc}
a_{0} b_{-}+a_{-} b_{0}+g_{e}\left(a_{0} a_{-}+b_{0} b_{-}\right) & 0 & 0 \\
0 & a_{0} c_{+}+a_{+} c_{0}+g_{e}\left(a_{0} a_{+}+c_{0} c_{+}\right) & 0 \\
0 & 0 & -c_{+} b_{-}+g_{e}\left(a_{-} a_{+}\right)
\end{array}\right)
$$

Note that both the ZFS tensor and the $g$-tensor of the spin Hamiltonian are diagonal in the molecular axes. This expression of the $\boldsymbol{g}$-tensor corresponds to the standard use of the spin Hamiltonian expressed in the molecular axes and $\chi_{-1}^{1}, \chi_{0}^{1}, \chi_{1}^{1}$, so these values are the ones to be introduced in simulation programs as the standard $g_{x}, g_{y}$, and $g_{z}$ values.

The effect of a magnetic field along the $z$-axis on the energy of the states is shown in Fig. 3c. Note that, although allowed EPR transitions could take place $\left(\Delta m_{s}= \pm 1\right)$, no EPR signal has been reported for standard microwave frequencies. The reason is that for the reported systems, the splitting between the ground $\psi_{0}$ and $\psi_{ \pm 1}$ in Fig. $3 \mathrm{c}$ is too large for X-band microwave photons $\left(h \nu \approx 0.3 \mathrm{~cm}^{-1}\right.$ ). Recently, high-field EPR $(\nu \approx 600 \mathrm{GHz})$ of Fe(IV)-corroles has been reported and values for parameter $D$ were found to be $14 \mathrm{~cm}^{-1}$ and $21 \mathrm{~cm}^{-1}$ for two different systems [4].

In Fig. 4, some graphs are collected that illustrate the dependence of the zerofield parameters $D$ and $E$ (Fig. 4a and b), and the $g^{F e}$-values (Fig. 4c) on the crystal-field parameters $\Delta$ and $V$. When the rhombic distortion parameter $V$ is 0 , then $E=0$ and just two g-values are observed $\left(g_{x}=g_{y}\right)$. This happens in case the iron ion has a fourfold axis of symmetry. The $D$ parameter and $g_{/ /}$are just slightly affected by changes in $V$, but $|E|$ increases with increasing $V$, and $g_{\perp}$ is split into $g_{x}$ and $g_{y}$ with $g_{x}>g_{y}$.

\subsection{Fe(IV)-Oxo Complex in General Symmetry: Directions of the g-tensor and Electronic Orbitals}

If the crystal-field potential includes contributions that do not correspond to the octahedral symmetry axes, which are the molecular axes (that is the $\mathrm{N}-\mathrm{Fe}-\mathrm{N}$ bonds and Fe-axial ligand axis), the eigenvectors of the crystal-field potential will not correspond with the $t_{2 g}$ orbitals, but, as they are a basis set for the subspace, they will be a linear combination of them: 

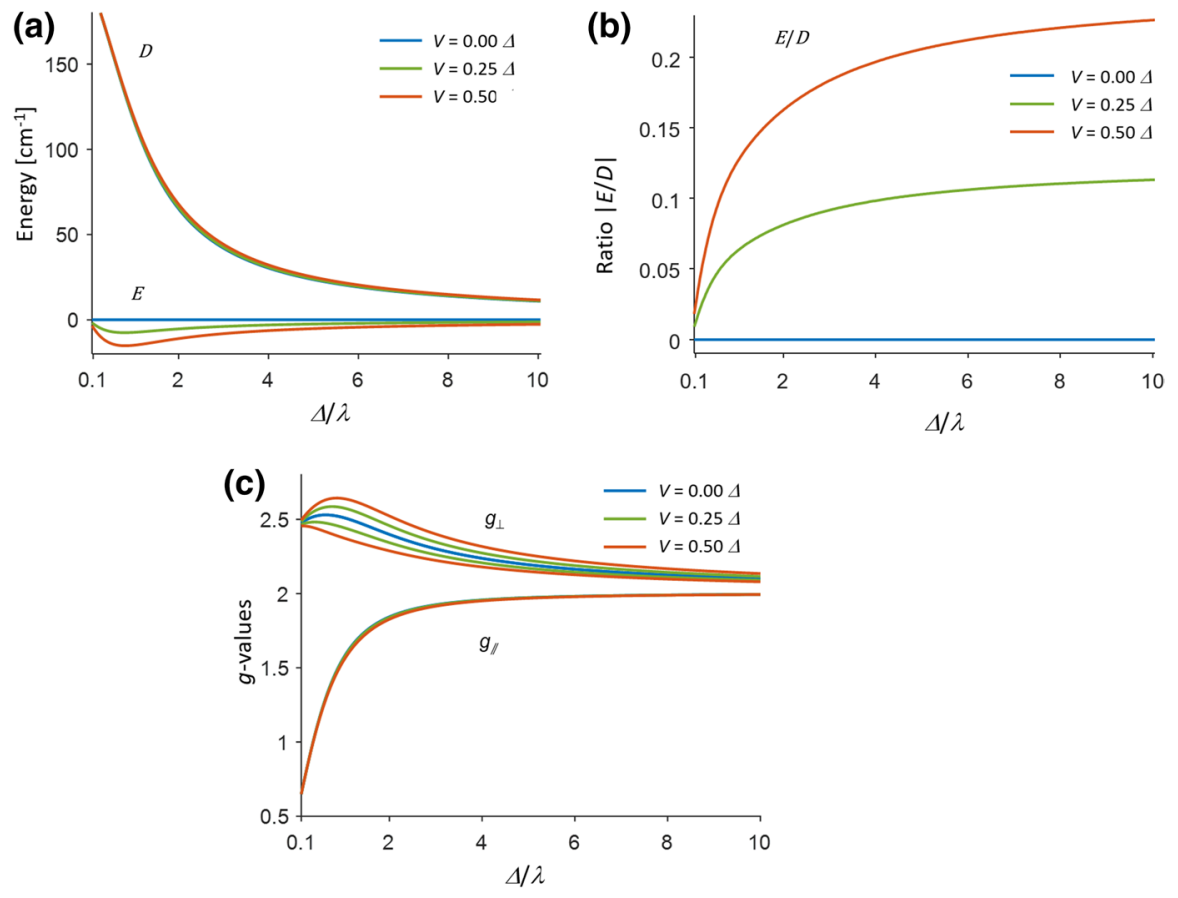

Fig. 4 a Dependence of $D$ and $E$ on $\Delta / \lambda$ and $V$. Note that for $V=0, E=0$ (blue line). b Dependence of the $|E / D|$ on $\Delta / \lambda$. For $V=0,|E / D|$ ratio is 0 (blue line). For higher rhombicity, the ratio monotonically increases. $\mathbf{c}$ Dependence of the $g$-values of Fe on the same parameters as before. Note that for $V>0$, the $g_{\perp}$ is separated into $g_{x}$ and $g_{y}$ with $g_{x}>g_{y}$ (color figure online)

$$
\left(\begin{array}{c}
\phi_{X} \\
\phi_{Y} \\
\phi_{Z}
\end{array}\right)=\Omega\left(\begin{array}{l}
d_{y z} \\
d_{x z} \\
d_{x y}
\end{array}\right)=\Omega\left(\begin{array}{l}
\varphi_{x} \\
\varphi_{y} \\
\varphi_{z}
\end{array}\right) .
$$

The relation between the two sets of states (low-symmetry eigenstates and d-orbitals) is given by a unitary matrix $\Omega$ that can be interpreted as a spatial rotation matrix [5,6]. $\Delta$ and $V$ can be defined in reference to the energy levels of the low-symmetry eigenstates [7]. If a new reference frame is introduced by rotating the original $(x, y$, and $z)$ axis system by $\Omega$, the matrix expression of the one-electron orbital angular momenta in the new $(X, Y, Z)$ axis system referred to in the rotated eigenstate basis set $\left(\phi_{X}, \phi_{Y}, \phi_{Z}\right)$ is the same as the angular momentum operators in the orthogonal axis system expressed in $\left(\varphi_{x}=d_{y z}, \varphi_{y}=d_{x z}, \varphi_{z}=d_{x y}\right)$ basis set [5, 6]:

$$
\left\langle\phi_{J}\left|\hat{\ell}_{k}\right| \phi_{I}\right\rangle=\left\langle\varphi_{j}\left|\hat{\ell}_{k}\right| \varphi_{i}\right\rangle .
$$

As a consequence of these relationships, it can be easily found that $(X, Y, Z)$ are the principal axes of the gyromagnetic tensor in the low-symmetry case [7]. In low-spin ferric heme systems, the EPR signal of the iron can be observed and the 


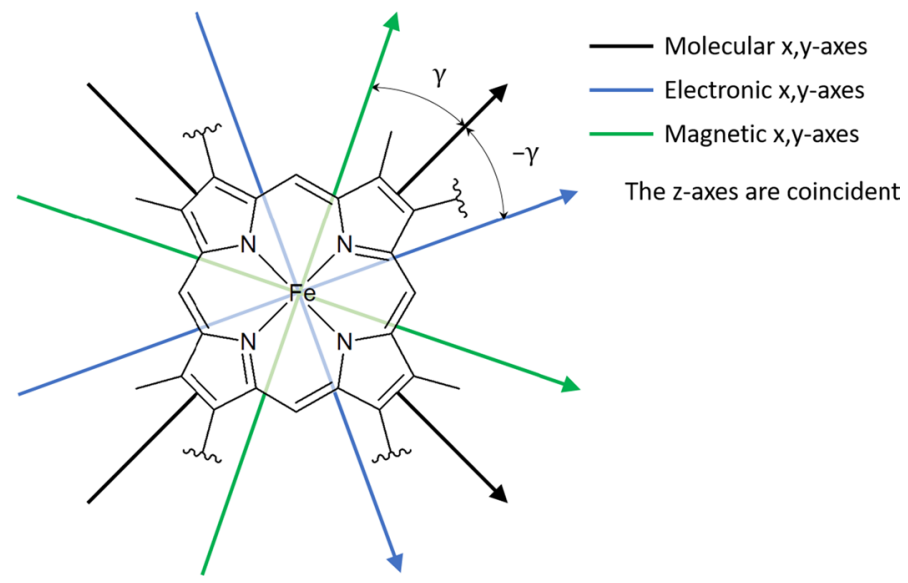

Fig. 5 Counter-rotation of the magnetic axes ( $\mathrm{g}$ and ZFS tensors) and the electronic axes (orientation of the semi-occupied orbitals) with respect to the molecular axes. All three axis-systems share the heme normal as $z$-axis

orientation of the gyromagnetic principal axes has been studied in detail. In these $d^{5}$ systems, it has been observed that the symmetry imposed by the heme plane is dominating and, therefore, the heme normal is a principal axis of the $g$-tensor, that is, $z$ coincides with $Z$. For ferryl systems, the symmetry of the heme site does not seem to be altered; moreover, binding of the oxo ligand along the $z$ molecular axis reinforces the symmetry along this direction. In this case, as for the low-spin ferric heme, the magnetic axes are rotated about the heme normal with respect to the molecular axes by an angle $\gamma$. Therefore:

$$
\Omega=\left(\begin{array}{ccc}
\cos \gamma & -\sin \gamma & 0 \\
\sin \gamma & \cos \gamma & 0 \\
0 & 0 & 1
\end{array}\right)
$$

And the corresponding eigenstates are then:

$$
\left(\begin{array}{l}
\phi_{X} \\
\phi_{Y} \\
\phi_{Z}
\end{array}\right)=\Omega\left(\begin{array}{l}
d_{y z} \\
d_{x z} \\
d_{x y}
\end{array}\right)=\left(\begin{array}{ccc}
\cos \gamma & -\sin \gamma & 0 \\
\sin \gamma & \cos \gamma & 0 \\
0 & 0 & 1
\end{array}\right)\left(\begin{array}{l}
d_{y z} \\
d_{x z} \\
d_{x y}
\end{array}\right)=\left(\begin{array}{c}
\cos \gamma d_{y z}-\sin \gamma d_{x z} \\
\sin \gamma d_{y z}+\cos \gamma d_{x z} \\
d_{x y}
\end{array}\right),
$$

which, taking into account the dependence of the $d$-orbitals on the polar angles [Eq. (3)], yields

$$
\left(\begin{array}{l}
\phi_{X} \\
\phi_{Y} \\
\phi_{Z}
\end{array}\right)=\left(\begin{array}{c}
\sqrt{\frac{15}{16 \pi}} \sin 2 \theta(\cos \gamma \sin \varphi-\sin \gamma \cos \varphi) \\
\sqrt{\frac{15}{16 \pi}} \sin 2 \theta(\sin \gamma \sin \varphi+\cos \gamma \cos \varphi) \\
d_{x y}
\end{array}\right)=\left(\begin{array}{c}
\sqrt{\frac{15}{16 \pi}} \sin 2 \theta \sin (\varphi-\gamma) \\
\sqrt{\frac{15}{16 \pi}} \sin 2 \theta \cos (\varphi-\gamma) \\
d_{x y}
\end{array}\right)=\left(\begin{array}{l}
d_{z y^{\prime}} \\
d_{z x^{\prime}} \\
d_{x y^{\prime}}
\end{array}\right) .
$$



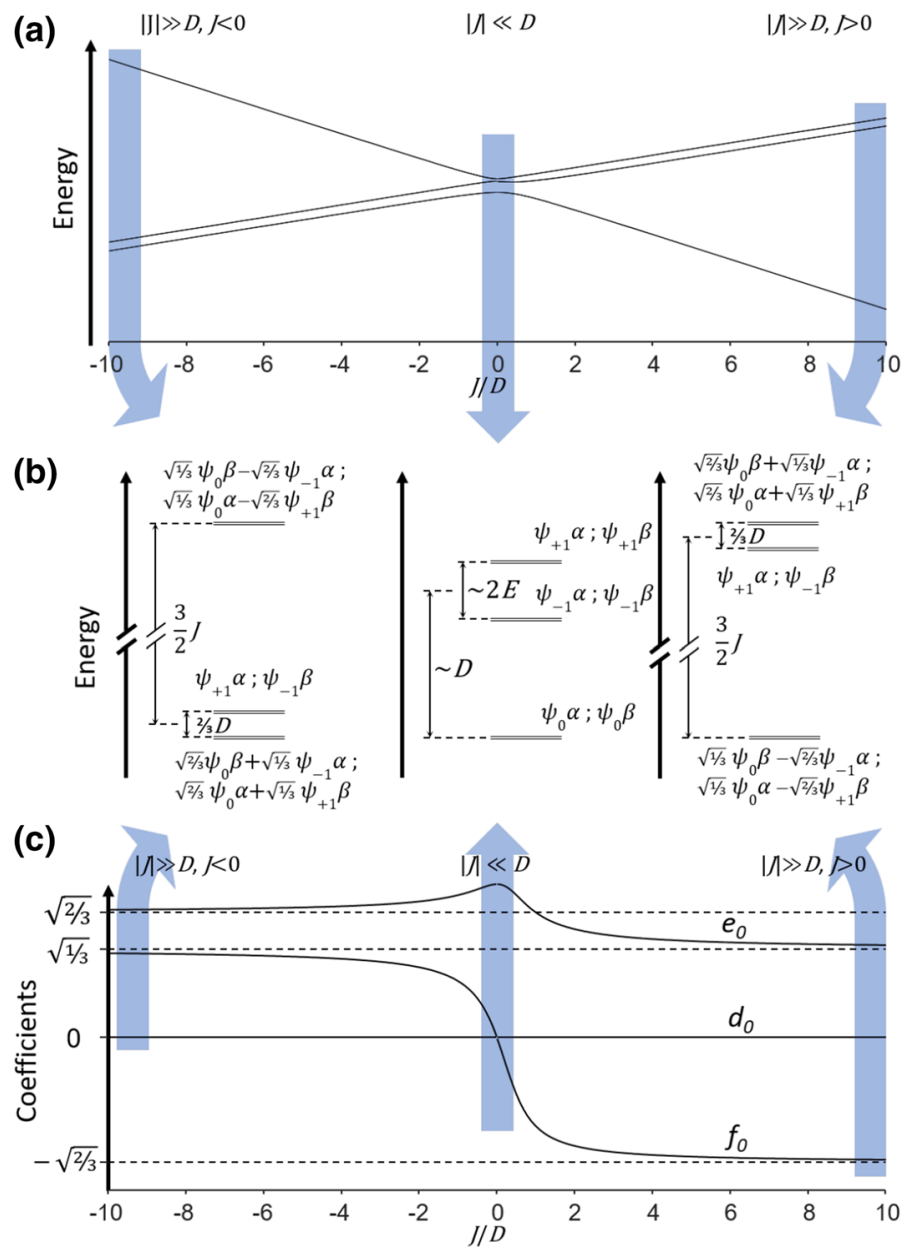

Fig. 6 Interaction of Fe(IV) $\left(S_{\mathrm{eff}}^{\mathrm{Fe}}=1\right)$ with a free radical $\left(S^{\prime}=1 / 2\right)$. a Energies of the three Kramers doublets as a function of the spin-spin isotropic coupling parameter in units of $D, J / D$. For the simulation $E=-0.1^{*} D$. b Eigenstates of the system in the case with $|J| \ll D$, and $|J| \gg D$, for $J>0$ and $J<0$. c Variation of the state coefficients $d_{0}, e_{0}$ and $f_{0}$ as a function of $J / D$. For the simulation, $E=0$

And therefore, one finds that the occupied orbitals in the low-symmetry case are rotated with respect to the standard octahedral orbitals by an angle $-\gamma$ about the axis perpendicular to the heme plane, whereas the magnetic axes are rotated by an angle $\gamma$ with respect to the molecular axes, see Fig. 5. This is the so-called counterrotation effect $[7,8]$, well studied for low-spin Fe(III), but that also applies to ferryl systems, with the particularity that two of these perpendicular orbitals are occupied, as summarized above. 


\subsection{Interaction Between Free Radical and Fe Moiety}

In Compound I, besides Fe(IV), there is an additional paramagnetic entity, a free radical $\left(S^{\prime}=1 / 2\right)$ that might be in a close-by location and therefore, a magnetic interaction between them is established. Due to the additional spin coordinates, the energy levels of the system are doubled with respect to the ones shown in Fig. 3 for the ferryl ground state. For the limiting case that the magnetic interaction is very weak or negligible, the two levels differing in the spin state of the interacting radical will be (almost) degenerate. If the magnetic interaction is strong, it completely rearranges the ground state of the iron, as shown in Fig. 6, to form new states minimizing the total energy, which depends on whether the interaction is ferromagnetic or antiferromagnetic. To calculate this energy, the spin-spin interaction term must be added to the spin Hamiltonian:

$$
\widehat{H}_{S S}^{S}=\widehat{\widehat{S_{\mathrm{eff}}^{F e}}} \widehat{\widehat{J S^{\prime}}}
$$

If we represent this term in the basis of states $\left\{\psi_{1} \alpha, \psi_{1} \beta, \psi_{0} \alpha, \psi_{0} \beta, \psi_{-1} \alpha, \psi_{-1} \beta\right\}$, assuming that the $\boldsymbol{J}$ tensor is also diagonal in the molecular axes:

$$
\hat{H}_{S S}^{S}=\hat{S}_{\text {eff }_{x}}^{\mathrm{Fe}} J_{x} \hat{S}_{x}^{\prime}+\hat{S}_{\text {eff }}^{\mathrm{Fe}} J_{y} \hat{S}_{y}^{\prime}+\hat{S}_{\mathrm{eff}_{z}}^{\mathrm{Fe}} J_{z} \hat{S}_{z}^{\prime}=\frac{\sqrt{2}}{4}\left(\begin{array}{ccccccc}
\sqrt{2} J_{z} & 0 & 0 & J_{x}-J_{y} & 0 & 0 \\
0 & -\sqrt{2} J_{z} & J_{x}+J_{y} & 0 & 0 & 0 \\
0 & J_{x}+J_{y} & 0 & 0 & 0 & J_{x}-J_{y} \\
J_{x}-J_{y} & 0 & 0 & 0 & J_{x}+J_{y} & 0 \\
0 & 0 & 0 & J_{x}+J_{y}-\sqrt{2} J_{z} & 0 \\
0 & 0 & J_{x}-J_{y} & 0 & 0 & \sqrt{2} J_{z}
\end{array}\right),
$$

rearranging the basis set, we find two identical $3 \times 3$ square matrices in the subspaces $\left\{\psi_{1} \alpha, \psi_{0} \beta, \psi_{-1} \alpha\right\}$ and $\left\{\psi_{-1} \beta, \psi_{0} \alpha, \psi_{1} \beta\right\}$ :

$$
\hat{H}_{Z F S}^{S}+\hat{H}_{S S}^{S}=\frac{\sqrt{2}}{4}\left(\begin{array}{cccccc}
\sqrt{2} J_{z}+\frac{4}{\sqrt{2}} \frac{D}{3} & J_{x}-J_{y} & -\frac{4}{\sqrt{2}} E & 0 & 0 & 0 \\
J_{x}-J_{y} & -\frac{4}{\sqrt{2}} \frac{2 D}{3} & J_{x}+J_{y} & 0 & 0 & 0 \\
-\frac{4}{\sqrt{2}} E & J_{x}+J_{y} & -\sqrt{2} J_{z}+\frac{4}{\sqrt{2}} \frac{D}{3} & 0 & 0 & 0 \\
0 & 0 & 0 & \sqrt{2} J_{z}+\frac{4}{\sqrt{2}} \frac{D}{3} & J_{x}-J_{y} & -\frac{4}{\sqrt{2}} E \\
0 & 0 & 0 & J_{x}-J_{y} & -\frac{4}{\sqrt{2}} \frac{2 D}{3} & J_{x}+J_{y} \\
0 & 0 & 0 & -\frac{4}{\sqrt{2}} E & J_{x}+J_{y} & -\sqrt{2} J_{z}+\frac{4}{\sqrt{2}} \frac{D}{3}
\end{array}\right) \text {. }
$$

As a result, for every energy level, there will be two eigenstates, the complete system has six eigenstates that associate to form three Kramers doublets that are well separated in energy (see Fig. 6). In many experimental cases, the spin-spin interaction tensor has been found to be isotropic, and therefore, orbital overlap, by means of an isotropic exchange interaction either positive (antiferromagnetic), or negative (ferromagnetic) has been postulated as the mechanism of interaction. For the other limiting case, i.e., a magnetic interaction that is large compared to the ZFS (spin-orbit coupling in Fe(IV)), one can find the energy of the Kramers doublets and 
the expression of the states by diagonalizing the $3 \times 3$ submatrices above treating $D$ and $E$ to first order (see Fig. 6).

For the intermediate cases between the two limiting cases, the two states for each of the three Kramers levels will be identical mixtures of the basis elements for each subspace. For example, for the ground doublet:

$$
\begin{aligned}
& \Psi_{+}=d_{0} \psi_{+1} \alpha+e_{0} \psi_{0} \beta+f_{0} \psi_{-1} \alpha \\
& \Psi_{-}=d_{0} \psi_{-1} \beta+e_{0} \psi_{0} \alpha+f_{0} \psi_{+1} \beta .
\end{aligned}
$$

The dependence of the energy of the doublets on the magnitude and sign of $J$ is shown in Fig. 6a, together with the expression for the eigenstates for the above-mentioned limiting cases (see Fig. 6b). The variation of the eigenstate coefficients of the ground doublet for increasing magnetic coupling is displayed in Fig. 6c. For the case of $|J| \ll D$, the coefficient $e_{0}$ is equal to 1 , while the other two coefficients are 0 .

When a magnetic field is applied, the spin Hamiltonian of the complete system must contain the electron Zeeman of the free radical, as well. Assuming that the radical has an isotropic $g$-factor equal to $g_{e}$ :

$$
\hat{H}^{S}=\hat{H}_{Z F S}+\widehat{\widehat{S_{\mathrm{eff}} \mathrm{Je}}} \widehat{\overrightarrow{S^{\prime}}}+\mu_{B} \vec{B}\left(g^{\mathrm{Fe}} \widehat{\widehat{S_{\mathrm{eff}}^{F e}}}+g_{e} \widehat{\overrightarrow{S^{\prime}}}\right) .
$$

Again, the energy of the EPR transitions at X-band $\left(\mathrm{h} \nu \sim 0.3 \mathrm{~cm}^{-1}\right)$ is too small to produce transitions between the states of the different doublets; however, EPR transitions can take place between the two states of these doublets. Since the EPR measurements must be performed at very low temperature due to the fast magnetic relaxation of the system, only the ground doublet $\left\{\Psi_{+}, \Psi_{-}\right\}$is populated and will, therefore, be the only one contributing to the EPR spectrum. Since the EPR spectrum is produced by a single transition between two levels, it can be represented in terms of an effective spin $S_{\text {eff }}^{\prime}=1 / 2$. In this case, the spin Hamiltonian will only contain the electron Zeeman term. Again, taking into account the expressions of the ground states above, the effective $\boldsymbol{g}$-tensor can be obtained calculating the effect of the electron Zeeman term on the ground doublet as a perturbation in the first order:

$$
\begin{aligned}
& \left\langle\Psi_{ \pm}\left|g_{x}^{F e} \widehat{S}_{\mathrm{eff}_{x}}^{F e}+g_{e} \widehat{S}_{x}^{\prime}\right| \Psi_{ \pm}\right\rangle=\left(2 \sqrt{2}\left(f_{0} e_{0}+e_{0} d_{0}\right) g_{x}+\left(2 f_{0} d_{0}+e_{0}^{2}\right) g_{e}\right)\left(\begin{array}{cc}
0 & 1 / 2 \\
1 / 2 & 0
\end{array}\right)=g_{x}^{\mathrm{eff}} \widehat{S}_{\mathrm{eff}_{x}^{\prime}}
\end{aligned}
$$

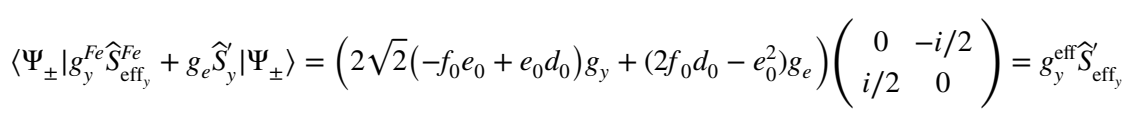

$$
\begin{aligned}
& \left\langle\Psi_{ \pm}\left|g_{z}^{F e} \widehat{S}_{\mathrm{eff}_{z}}^{F e}+g_{e} \widehat{S}_{z}^{\prime}\right| \Psi_{ \pm}\right\rangle=\left(2\left(d_{0}^{2}-f_{0}^{2}\right) g_{z}+\left(d_{0}^{2}-e_{0}^{2}+f_{0}^{2}\right) g_{e}\right)\left(\begin{array}{cc}
1 / 2 & 0 \\
0 & -1 / 2
\end{array}\right)=g_{z}^{\text {eff }^{\prime} \widehat{S}_{\mathrm{eff}_{z}^{\prime}}}
\end{aligned}
$$

Note that, again, for an isotropic spin-spin interaction, the $\boldsymbol{g}$-tensor is diagonal in the axes of the crystal field. Figure 7 depicts the effective $g$-values of the system 
(a)

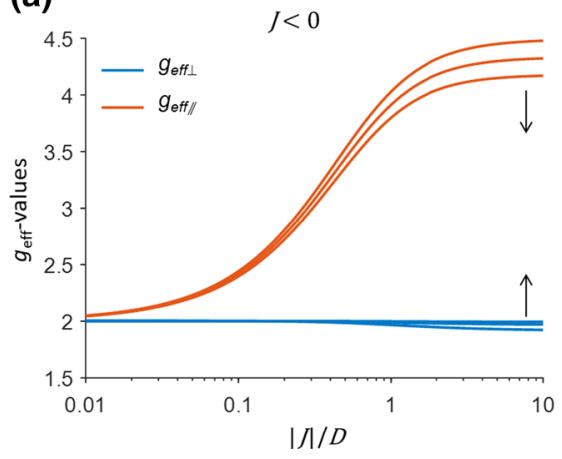

(b)

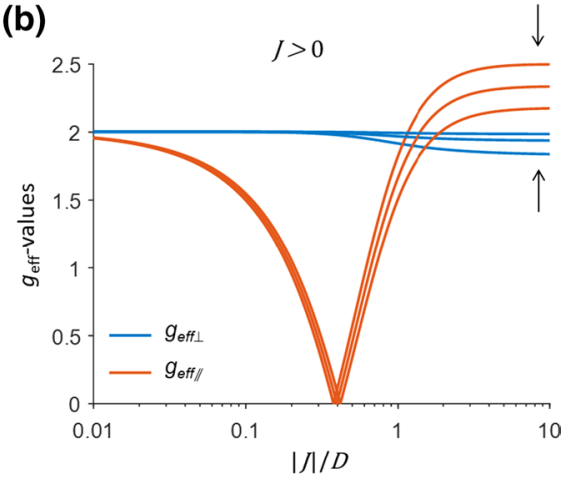

Fig. 7 Effective $g$-values for Compound I as function of $|J| / D$. The values used for the simulation are $V \approx$ $0, \lambda=400 \mathrm{~cm}^{-1}$ and $\Delta=900,1500,3000 \mathrm{~cm}^{-1}$. The corresponding $D$-values of the iron are, respectively, $57.6,32.7$, and $15.0 \mathrm{~cm}^{-1}$. The arrows indicate the movement of the graphs by increasing $\Delta$. The sign of $J$ changes from negative (ferromagnetic) (a) to positive (antiferromagnetic) (b)

as a function of the isotropic $J$-coupling (the case of $J<0$ in Fig. 7.a; and $J>0$ in Fig. $7 \mathrm{~b})$ for an axial system $(V=0)$ and different values of $\Delta$ in the range of the ones found for Cpd-I in heme catalysts. As was pointed out before [9], for both, antiferro- and ferro- magnetic interaction between the iron and the radical species, the feature corresponding to $g_{z}$ along the heme normal is invariably close to $g_{\text {eff }}=2$, while the $g$-values corresponding to directions in the heme plane depend strongly on the strength of the magnetic coupling. Obviously, both are close to $g_{\text {eff }}=2$ in the limit of weak coupling, but as the magnetic interaction gets stronger, the ferromagnetic system $(J<0)$ features $g$-values larger than 2 (lower fields), and the antiferromagnetically $(J>0)$ coupled systems have $g$-values lower than 2 , reaching a minimum $g$-value of zero and increasing again to end up with values larger than 2 for the strong coupling case.

In the strong coupling limit, upon increasing $\Delta$, the $g_{\text {eff// }}$ are getting closer to $g_{e}$.

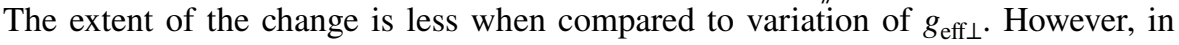
both cases, the $g_{e}$ value is never reached and the $g_{\text {eff // }}$ and $g_{\text {eff } \perp}$ approach an asymptote that depends on $\Delta$. When an anisotropic $J$ is used for the simulation, the $g$-values are separated into three components.

\subsection{Orbach relaxation processes}

The $g$-values are not the only EPR feature of the free radical that is altered by the interaction with the $S=1$ iron moiety. Also the relaxation times are severely shortened due to the much stronger coupling of the iron with the vibrations of the lattice [10]. At very low temperatures, one-phonon-mediated relaxation processes (direct process) dominate the evolution of the relaxation rate with temperature. As the temperature is increased two-phonon Orbach relaxation mechanisms take over [11]. These mechanisms involve the absorption of a phonon to an excited state and emission of another phonon back to the ground doublet (see Fig. 8a). Both processes have a characteristic dependence with temperature (see Eq. 27). 
(a)

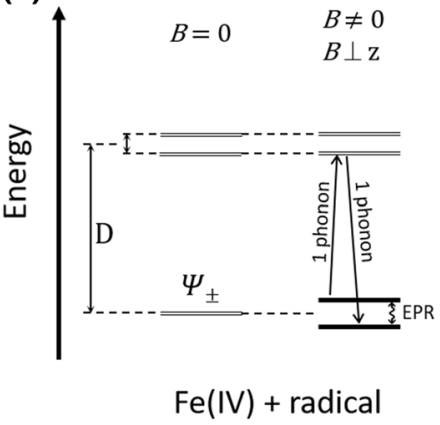

(b)

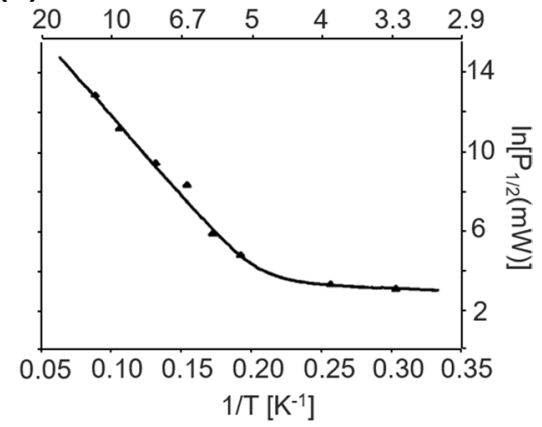

Fig. 8 a Energy-level diagram of low spin Fe(IV) in an exchanged-coupled Cpd-I (with small rhombicity). Left: effect of the zero-field splitting on the energy levels, in a zero magnetic field. Right: the applied magnetic field splits the Kramers doublets. For clarity, just the lower doublet (the only one that is populated at EPR temperatures) is separated in the figure. The arrows indicate the phonon absorption and emission typical of the Orbach spin-lattice relaxation process. The routinely measured EPR transition is between the two energy levels of the lowest Kramers doublet. b Half-saturation power $\mathrm{P}_{1 / 2}$ as a function of temperature measured for CPO-I. Adapted with permission from [9]. Copyright (1984) American Chemical Society. The lineal part of the solid line at higher temperature indicates that for $\mathrm{T}>5 \mathrm{~K}$ the spin-lattice relaxation is dominated by Orbach transitions to an excited Kramers doublet

Interestingly, Orbach processes depend on the energy separation between the excited states and the ground state. Therefore, the experimental determination of spin-lattice relaxation times $\left(T_{1}\right)$ through the study of CW-EPR saturation curves, pulse saturation recovery, or inversion recovery experiments as a function of temperature, can yield the energy separation between the ground Kramers doublet and the next one $(\Delta E)$, corresponding to the first excited iron energy level (see Fig. 8a):

$$
\frac{1}{T_{1}}=A T+B\left(e^{\Delta E / T}-1\right)^{-1},
$$

where $A$ and $B$ are fitting parameters. Relaxation studies as a function of temperature have been used to univocally determine the $D$-values of heme proteins via EPR [12-15]. In Fig. 8b, the spin-lattice relaxation rate obtained for Cpd-I of the enzyme chloroperoxidase [9] is plotted versus the inverse temperature and fitted to Eq. 27, containing the sum of the direct process and Orbach process relaxation rates. The magnitude of the zero-field splitting can be obtained using this method. The advantages of this method are its sensitivity and accuracy, but it needs frozen solutions with a tight temperature control $[12,13]$.

The general frame of the spin-coupling model described in this paragraph will be exemplified below using the main biocatalysts for which Compound I was isolated and measured by CW EPR. Using the published EPR spectra and depending on whether there are estimations of the zero-field parameters (either, by high-field EPR, the study of relaxation times or Mössbauer experiments), the components of the spin-spin coupling will be adjusted to fit the experimental result. 

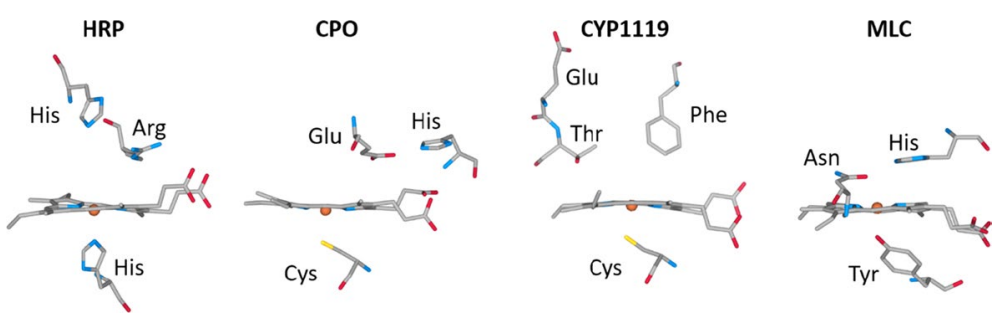

Fig. 9 Different key amino acids in the proximal and distal offside of the heme pocket for four heme proteins discussed in Sect. 3. The active site is taken from the resting state, with Fe(III). Color code: gray for carbon, blue for nitrogen, red for oxygen, yellow for sulfur, and brown for iron. The Hydrogen atoms and water molecules are omitted. The proximal ligand is located at the bottom side of the heme; on the other side, the sixth ligand (usually a water molecule) is omitted; and the main amino acids responsible for the formation of Cpd-I are reported. The HRP has a histidine as proximal ligand and the polar cavity on the distal side. CPO and P450 have the same proximal ligand, a cysteine. However, CPO polar distal cavity resembles the one of peroxidases, rather than the more non-polar $\mathrm{P} 450$. Catalase has a tyrosine as proximal ligand, and shares with HRP a histidine in the distal pocket that works together with another polar residue to perform the acid-base catalytic mechanism that allows the formation of Cpd-I. Figures were generated with CCP4mg [16]. The PBD files used are from left to right: 1ATJ, 1CPO, 1IO7, and 1GWE (color figure online)

\section{Selected Overview of EPR Studies of Compound I in Heme Enzymes}

In this section, we are going to review the published data from selected enzymes for which the EPR spectrum of Cpd-I has been reported. The validity of the model detailed in the previous section will be illustrated by simulating the very different reported spectra of four different enzymes performing different chemistry with different axial ligations (see Fig. 9).

\subsection{Horseradish Peroxidase}

Horseradish peroxidase (HRP) has been known for more than a century and it has been extensively studied using different techniques. Most commonly, this enzyme uses hydrogen peroxide to oxidize a wide range of organic and inorganic compounds. Its Compound I intermediate (HRP-I) is relatively stable and it has thus been used as a model system. Its reactivity has been widely exploited for commercial uses, like clinical diagnostic kits and immunoassays [17]. The proximal axial ligation side of the heme iron is occupied by an imidazole nitrogen from a histidine side chain, and the distal coordination side changes during the reaction sequence. The catalytic cycle starts with the binding of hydrogen peroxide to the distal side of the heme iron and the subsequent cleavage and release of a water molecule leaving an oxygen atom bound to the iron with a formal charge -2 , which leads to the formation of Cpd-I. The proton transfer needed for this process is supposed to be assisted by a distal histidine residue present in the heme pocket. The intermediate returns to the resting state through oxidation of two molecules of substrate, as shown in Fig. 10a [17, 18]. 
(a)

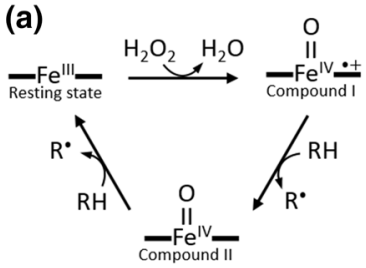

(c)

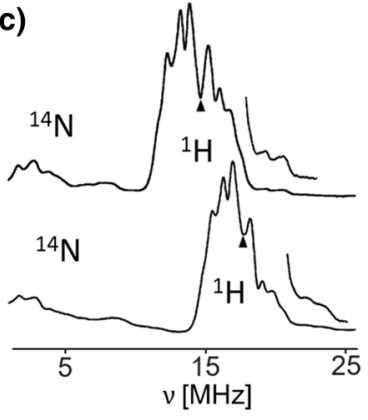

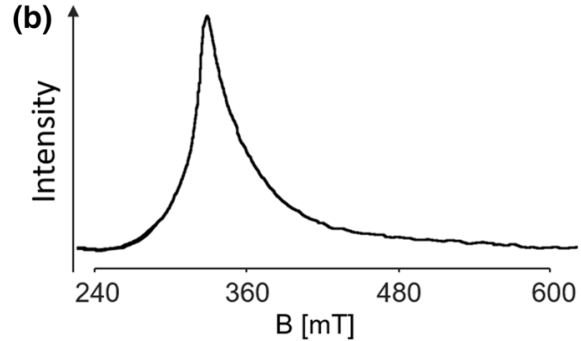

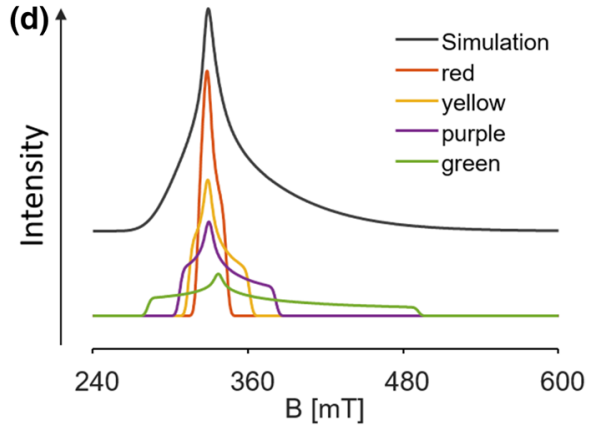

Fig. 10 a Schematic reaction cycle of horseradish peroxidase. The resting state first reacts with hydrogen peroxide to form Cpd-I which in turn is converted to Compound II by oxidizing a substrate molecule and back to the resting state after oxidizing a second molecule. b CW-EPR spectrum of HRP-I measured under rapid passage conditions featuring a sharp line at $g \approx 2$ and broad wings at both sides of the central feature. Reproduced with permission from Ref. [15]. c ENDOR spectra taken at $g=2.0$, $9.60 \mathrm{GHz}$ (top) and $11.66 \mathrm{GHz}$ (bottom). $\boldsymbol{\Delta}$ Indicates the free proton Larmor frequency. Reproduced with permission from ref. [27]. d Upper spectrum: simulation performed with the EPR simulation toolbox EasySpin [28] using a Gaussian distribution for the exchange interaction. The exchange tensor was kept axial, traceless, and proportional to $\boldsymbol{J}=[4,-2,-2] \mathrm{K}=[2.8,-1.4,-1.4] \mathrm{cm}^{-1}$, which was the center of the distribution with an FWHM $=[3.1,1.6,1.6] \mathrm{cm}^{-1}$. The spectra below show individual contributions within the distribution for which the exchange tensor, expressed in $\mathrm{cm}^{-1}$, and weights (w) were: red: $\boldsymbol{J}=[0.5,-0.3,-0.3], \mathrm{w}=0.19$; yellow $\boldsymbol{J}=[1.1,-0.5,-0.5], \mathrm{w}=0.36$; purple: $\boldsymbol{J}=[1.6,-0.8,-0.8], \mathrm{w}$ $=0.58$; green: $\boldsymbol{J}=[3.9,-1.9,-1.9], \mathrm{w}=0.60$. The rest of the spin Hamiltonian parameters used for all contributions are: $g_{\text {eff }}^{F e}=(2.21,2.21,1.97), D=26 \mathrm{~cm}^{-1}, E=0$, corresponding to crystal-field parameters $\Delta=1830 \mathrm{~cm}^{-1}, V=0$ taking a spin-orbit coupling constant of $\lambda=400 \mathrm{~cm}^{-1}$. The simulation script is available as complementary information (color figure online)

The crystal structure of HRP-I has been obtained by X-ray crystallography. The $\mathrm{Fe}-\mathrm{O}$ distance obtained is $1.7 \AA$, that is indicative of an unprotonated double bond $\mathrm{Fe}(\mathrm{IV})=\mathrm{O}$. The distance of the iron from the proximal histidine is $2.14 \AA$ [19-21]. Additionally, EXAFS studies have also been carried out yielding an $\mathrm{Fe}=\mathrm{O}$ distance of $1.67 \AA$, in accordance with the crystallographic structure [22].

Mössbauer spectra of HRP-I show a decrement of the isomeric shift with respect to the resting enzyme, revealing a loss of d-charge density due to a metal-based oxidation and proving the formation of an $\mathrm{Fe}(\mathrm{IV})=\mathrm{O}$ species in the low-spin $3 d^{4}$ configuration $(S=1)$. The zero-field splitting of HRP-I was first obtained from Mössbauer studies, obtaining $D=32 \mathrm{~K}\left(22 \mathrm{~cm}^{-1}\right)$ [15]. 
The first CW-EPR experiment of HRP-I that showed the complete spectrum was recorded at $3.4 \mathrm{~K}$ under rapid adiabatic passage, which yields an absorption spectrum (see Fig. 10b). It consists of a feature located around $g \approx 2$ accompanied by long and broad wings extended in a window of about $400 \mathrm{mT}$ [15]. Normal, slowpassage measuring conditions are not optimal to detect broad signals; actually, the previous reports on EPR signals of HRP-I overlooked a large majority of the signal merged with the baseline obtaining only an integrated intensity of about $1 \%$ of the total number of spins and thus largely underestimating the amount of Cpd-I formation [23].

The relaxation time $\left(T_{1}\right)$ of the radical (since the spin-spin interaction is small, one can consider the sharp spectral feature as being mostly due to the radical) is drastically shortened due to the interaction with the iron center and, therefore, low temperatures are generally needed for EPR detection of Cpd-I. As explained above, the relaxation times are heavily influenced by the electronic properties of $\mathrm{Fe}(\mathrm{IV})$ and the study of the evolution of $T_{1}$ with temperature lead to an estimation for the zerofield splitting parameter $D$ of the oxo-iron of $36.8 \pm 2.5 \mathrm{~K}\left(26 \pm 1.7 \mathrm{~cm}^{-1}\right)$, assuming $E=0$ [12], in agreement with Mössbauer estimations (see Table 1 for summary of parameters).

The main feature of the spectrum is assigned to the $g_{\mathrm{z}}$ component (along the heme normal) of the $\boldsymbol{g}_{\text {eff }}$ tensor which, as previously shown in Fig. 7, stays at $g \approx 2$ and is quite insensitive to crystal-field parameters or spin-spin interactions. Small changes in the environment of the paramagnetic center lead to changes in the exchange coupling constant, that in turn are reflected in a distribution of $g_{\mathrm{x}}$ and $g_{\mathrm{y}}$ but leaving $g_{\mathrm{z}}$ almost unchanged (see Fig. 7). Therefore, the broad wings of the spectrum correspond to other microenvironments of the paramagnetic center, and different $J / D$-values. Since there are signals at both sides of the main feature, it seems clear that some of the orientations would correspond to positive and others to negative magnetic interactions. The authors simulated the spectrum with an anisotropic and small coupling tensor $\boldsymbol{J}=(2.8,-1.4,-1.4) \mathrm{cm}^{-1}=(4,-2,-2) \mathrm{K}$. We have also simulated the spectrum (see Fig. 10d) with the parameters reported in the figure caption. The authors included Gaussian distributions of the coupling values to account for the strong broadening found in the spectrum. It should be noted that because $|J| / D \approx 0.1$, the magnetic interaction between the paramagnetic centers is small and, therefore, the total spin is not a good quantum number [15, 24]. Mössbauer data confirmed this point though indications of a weak coupling of the iron with the porphyrin $\pi$-cation radical [25].

The detection of weak hyperfine interactions of the unpaired electron with the ${ }^{17} \mathrm{O}$ nucleus of Cpd-I using ENDOR and isotopically enriched $\mathrm{H}_{2}{ }^{17} \mathrm{O}_{2}$ allowed to show that, in this species, an oxygen atom is bound to the iron [26]. Additionally, ENDOR findings [27] were crucial to associate the broad spectrum of HRP-I to a porphyrin $\pi$-cation radical. Proton and ${ }^{14} \mathrm{~N}$ ENDOR (see Fig. 10c) revealed that the dominant state is an $A_{2 u}$-like state with large spin densities on porphyrin nitrogens and $\beta$-carbons. Deuterium labeling showed that the proton ENDOR of an HRP-I sample derives from structural protons of the porphyrin macrocycle [27].

Both findings, the small value of $J$ and the porphyrin radical having a $A_{2 u}$ character, have been at first considered by Schultz et al. as proof that there is no overlap 


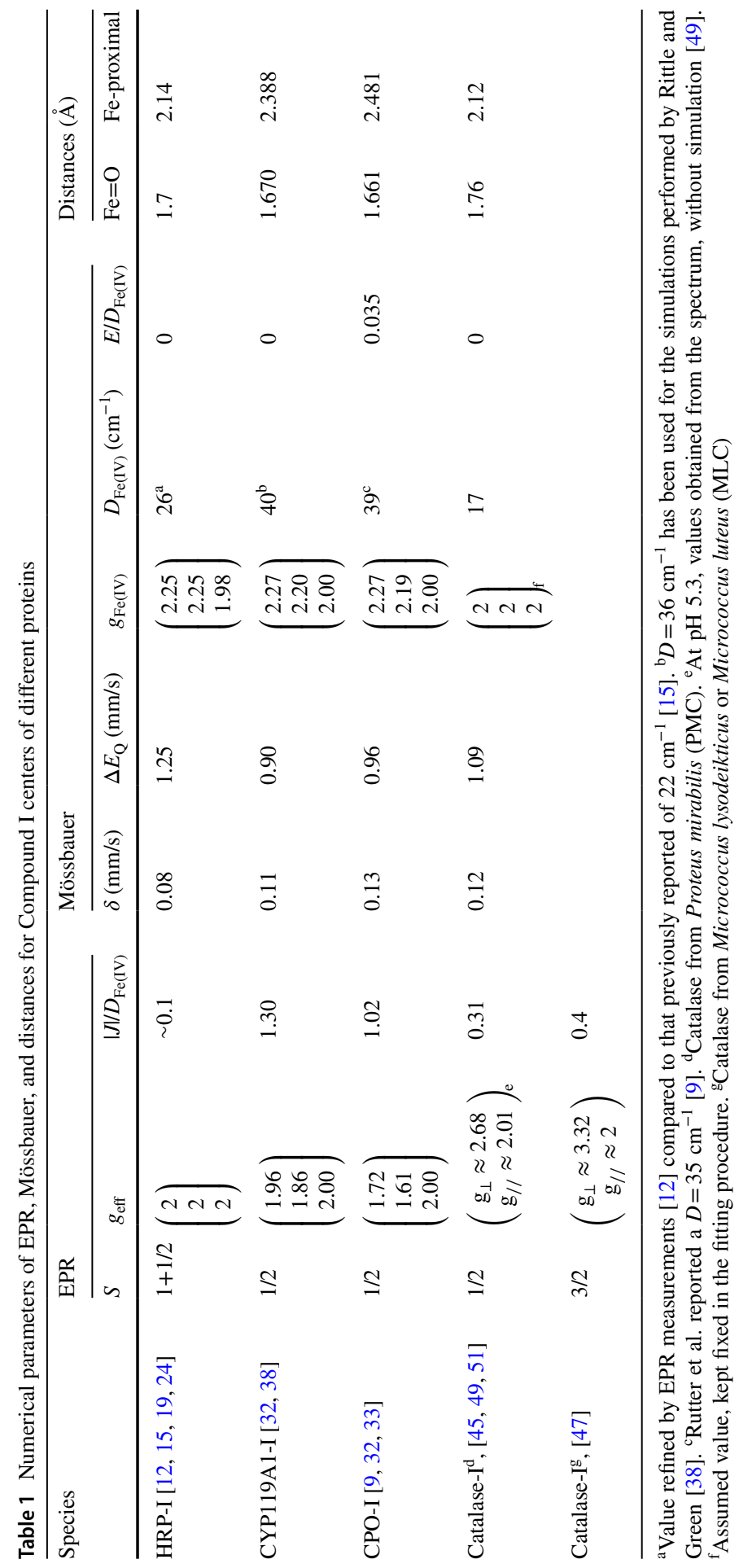


between the unpaired electrons in the $3 d$ iron orbitals and the porphyrin radical and they interpreted the interaction as a though-space magnetic interaction [15]. Later, the same authors state that the dipole-dipole interaction is too small to account for the reported $J$-values and that a contribution from the orbital overlap is needed [24]. In that respect, information about orientation of the iron semi-occupied orbitals would be appropriate, but there is no such information available at present.

\subsection{Chloroperoxidase}

The next example is a family of versatile proteins, chloroperoxidases (CPOs). Their name comes from the fact that they catalyze the chlorination of organic compounds using $\mathrm{H}_{2} \mathrm{O}_{2}$, organic hydroperoxides, or peracids as an electron acceptor. CPO is the most versatile haloperoxidase in the heme protein family. In addition to catalyzing halogenation reactions, CPO possesses a broad spectrum of oxidative activities, including those characteristics of heme peroxidases, catalases, and the P450 cytochromes (see below). Additionally, it shares with horseradish peroxidase and other members of the plant peroxidase family the ability to catalyze one and two electron peroxidations [29-31]. This enzyme is a secreted protein, therefore far more stable, and more importantly only requires $\mathrm{H}_{2} \mathrm{O}_{2}$, organic hydroperoxides, or peracids for its activation, in contrast with P450 (see next paragraph). The schematic cycle of the enzyme is shown in Fig. 11a for a typical chlorination reaction.

The active site of this enzyme is different from the one of HRP in that the proximal heme iron is coordinated to the sulfur atom of a cysteine side chain. On the distal side, the acid residue required to cleave the peroxide $\mathrm{O}-\mathrm{O}$ bond is glutamic acid instead of the histidine in HRP. No crystal structure of Chloroperoxidase Cpd-I (CPO-I) have been obtained until now, but EXAFS measurements of the one from Caldariomyces fumago have been performed obtaining average distances of $1.661 \AA$ and $2.481 \AA$ for $\mathrm{Fe}-\mathrm{O}$ and proximal Cys-Fe, respectively. The short $\mathrm{Fe}-\mathrm{O}$ distance is in accordance with a $\mathrm{Fe}=\mathrm{O}$ double bond $[32,33]$.

The CW-EPR spectrum of CPO-I was first obtained at $3 \mathrm{~K}$ measuring in the dispersion mode under rapid passage conditions [9, 34], which, as discussed above, favors the detection of broad signals and leads to an absorption spectrum. In addition to Cpd-I, the sample contained other species like the resting-state enzyme and an organic-based free radical, whose spectra were separated by performing saturation studies at different temperatures. The spectrum is quite different from the described HRP-I, it spans about $120 \mathrm{mT}$ in the spectrum and has two features (see Fig. 11b) one corresponding to $g_{/ /} \approx 2$ and the other at a value of the magnetic field corresponding to $g_{\perp} \approx 1.73$. In the same study, the microwave power to half saturate the spectrum $\left(P_{1 / 2}\right)$ was determined as a function of the temperature to obtain the temperature dependence of the spin-lattice relaxation time $T_{1}$ and the energy of the next Kramers doublet, needed to derive the crystal-field parameter $\Delta$ and the zero-field splitting parameter $D$. These values are in agreement with the ones found by variable-temperature Mössbauer spectroscopy, $D=36 \mathrm{~cm}^{-1}$ (52 K) [9], $39 \mathrm{~cm}^{-1}$ (56 K) [32]. The rest of Mössbauer parameters are summarized in Table 1 and are consistent with a ferryl heme state. 
(a)

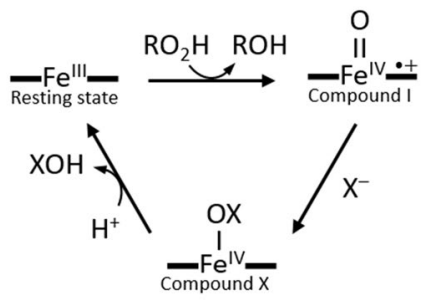

(c)

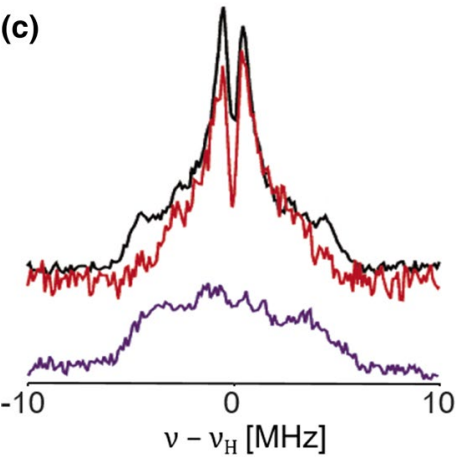

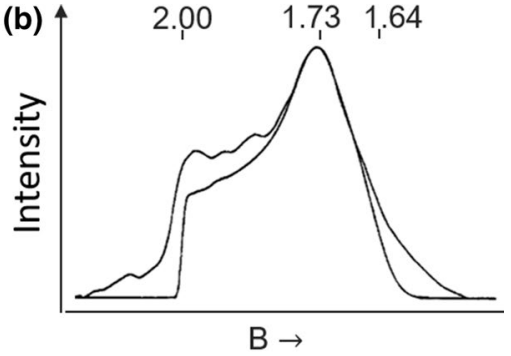

(d)

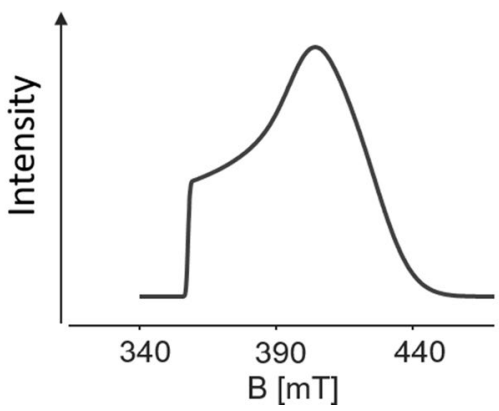

Fig. 11 a Reaction cycle of CPO. The resting state reacts with a peroxide to form Compound I, that, in turn, reacts with chloride or bromide to form Compound X, able to halogenate the substrate. b Absorption EPR spectrum of CPO-I taken at $3 \mathrm{~K}$ (upper trace) and its simulations (bottom trace). $g$-Values reported in the image. Adapted with permission from [9]. Copyright (1984) American Chemical Society. c Pulsed Q-band ENDOR taken at $g=1.75$. Upper traces: Stochastic Davies ${ }^{1} \mathrm{H}$ ENDOR in water (black) and $\mathrm{D}_{2} \mathrm{O}$ (red). Down trace (scaled): Mims ${ }^{2} \mathrm{H}$ ENDOR in $\mathrm{D}_{2} \mathrm{O}$. The zero-frequency corresponds to the proton Larmor frequency. Adapted with permission from [35]. Copyright (2006) American Chemical Society. d Simulation performed with EasySpin using a Gaussian distribution of isotropic exchange parameters centered at $J=39.9 \mathrm{~cm}^{-1}$ and FWHM of $2.1 \mathrm{~cm}^{-1}$. The rest of parameters for the simulations are: $g_{\text {eff }}^{\mathrm{Fe}}=(2.30,2.27,1.93), D=39.1 \mathrm{~cm}^{-1}, E=-1.37 \mathrm{~cm}^{-1}, J / D=1.02,|E / D|=0.035$ corresponding to crystal-field parameters $\Delta=1280 \mathrm{~cm}^{-1}, V=120 \mathrm{~cm}^{-1}$ taking a spin-orbit coupling constant $\lambda=400 \mathrm{~cm}^{-1}$. Please note the $g_{\text {rad }}$ used is 2.08 and not that one of the free electron. The script used for the simulation is available as supplementary information

With the value of $\Delta$ (or $D$ ), and recognizing a practically axial symmetry of the spectrum, it is possible to obtain the exchange interaction parameters and simulate the spectrum using the theory summarized in the previous paragraph. The spectrum has been successfully simulated either in the effective spin $S=1 / 2$ representation or using the exchange-coupled spin Hamiltonian, assuming a practically isotropic exchange parameter $J$ and including a Gaussian distribution about the principal axes values to model the line width. The value for $J$ was very similar to the one obtained for $D, J / D=1.02$, that is, a strong zero-field splitting and a strong antiferromagnetic coupling are found in CPO-I [9]. Also the Mössbauer spectra are in agreement with this finding as they show a dependence of the line intensities on the direction of the applied magnetic field, indicating that the iron nucleus is associated with the EPR 
signal in contrast to the Mössbauer spectra of HRP-I which show a much weaker magnetic interaction, leading to broadening of the quadrupole doublet [9].

Proton CW-ENDOR and pulsed-ENDOR studies of Cpd-I from CPO have been reported [35] and are shown in Fig. 11c. The ENDOR spectrum of CPO-I in $\mathrm{H}_{2} \mathrm{O}$ shows a number of doublets with maximum hyperfine coupling $\sim 10 \mathrm{MHz}$. When the spectrum is recorded in $\mathrm{D}_{2} \mathrm{O}$ buffer, the most strongly coupled peaks are lost, owing to exchangeable proton(s). This observation is confirmed by the ${ }^{2} \mathrm{H}$ ENDOR signal with the corresponding coupling. Thus, the other doublets are from protons of heme and/or proximal ligand with a maximum coupling constant of $\sim 6 \mathrm{MHz}$. This study leads to the conclusion that the radical is located predominantly on the porphyrin, with delocalization to sulfur just to a minor extent (maximum value for the sulfur spin density $\approx 0.23$ ). However, a $J / D$-value of 1.6 was used to make the calculations. The authors indicate that further ${ }^{1,2} \mathrm{H}$ and ${ }^{14} \mathrm{~N}$ studies should be carried out to better understand the CPO-I active site.

The spectrum reported in Fig. $11 \mathrm{~b}$ was simulated by the authors with an almost isotropic coupling tensor $\boldsymbol{J}=(36,36,37) \mathrm{cm}^{-1}=(52,52,54) \mathrm{K}$, a small rhombicity $E / D=0.035$ and a $g_{\text {rad }}=2.08$. We have also simulated the spectrum (see Fig. 11d) with the parameters reported in the figure caption, using an isotropic $J$.

\subsection{P450 Enzymes}

Important enzymes assumed to perform catalysis via Cpd-I are the members of the cytochrome P450 (P450) monooxygenase superfamily. They catalyze the selective oxy functionalization of many organic substrates under mild and environmentally friendly conditions, and some of them also perform peroxidase and peroxygenase reactions, like the versatile CPOs. However, unlike CPOs, some members of the P450 family are able to catalyze the terminal oxygenation of alkanes. Their catalytic cycle, schematically depicted in Fig. 12a, uses molecular oxygen and electrons coming ultimately from NADH and provided through physiological partner proteins to oxygenate the substrate. Owing to their requirement for costly co-substrates and auxiliary enzymes, among other reasons, industrial applications of these versatile biocatalysts mainly focus on the production of drug metabolites, pharmaceutical products, and some specialty chemicals. The first two electron transfers of the cycle are the rate-determining steps of the reaction whereas the formation of P450-I (P450 Cpd-I) and the formation of the product are much faster. Therefore, it is very hard to accumulate the reactive intermediate in the natural cycle. Nevertheless, ferric P450 $\left(\mathrm{Fe}^{\mathrm{III}}\right)$ can react with oxotransfer agents, peroxy compounds, and hydrogen peroxide to generate the Cpd-I via the peroxide shunt pathway (see Fig. 12a) [31].

Like in CPOs, the iron is axially coordinated by the sulfur atom of a cysteine residue in P450 enzymes. The crystal structure for an intermediate of P450cam was obtained at a resolution of $1.9 \AA$ by radiolytically reducing the ferrous oxy form of the enzyme during X-ray crystallography [36]. However, the intermediate obtained with this reduction procedure has not been identified univocally as Cpd-I. The distances obtained for this putative Cpd-I are about $1.65 \AA$ for the $\mathrm{Fe}=\mathrm{O}$ bond and $2.27 \AA$ for the proximal Cys-Fe [20, 37]. In the case of CYP119, distances have 

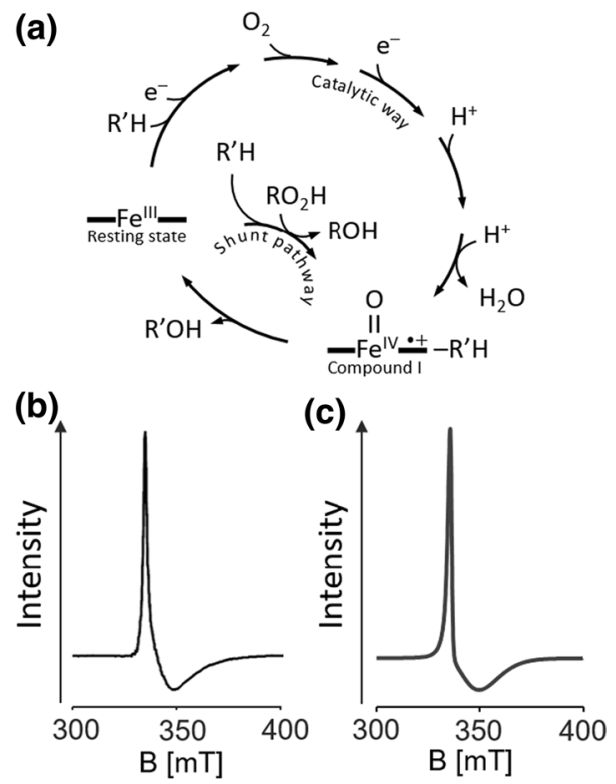

Fig. 12 a Reaction cycle of P450. The scheme reported is a simplified version of the entire cycle. The attention here is mainly focused on the shunt pathway; the interested reader is referred to [42]. $\mathrm{NAD}(\mathrm{P}) \mathrm{H}$ are the source of $\mathrm{e}^{-}$and $\mathrm{H}^{+}$. The peroxide shunt pathway is particularly important, because it is usually used to produce $\mathrm{P} 450-\mathrm{I}$. The resting state can react with a peroxide $\left(\mathrm{RO}_{2} \mathrm{H}\right)$ and a substrate molecule $\left(\mathrm{R}^{\prime} \mathrm{H}\right)$ to form the substrate-Compound I complex. After the reaction of the substrate, the resting state is regenerated and the product is released. b EPR spectrum of CYP119-I taken at 7.5 K, adapted from [38]. Reprinted with permission from AAAS. c. The simulation was performed with EasySpin using a Gaussian distribution of the isotropic exchange parameter $J=45.2 \mathrm{~cm}^{-1}$ with FWHM of $5.9 \mathrm{~cm}^{-1}$. Other parameters for the simulation: $\boldsymbol{g}_{\text {eff }}^{\mathrm{Fe}}=(2.27,2.27,1.94), D=36.2 \mathrm{~cm}^{-1}, E=0$ (corresponding to $\Delta=1370 \mathrm{~cm}^{-1}, V=0$ and $\left.\lambda=400 \mathrm{~cm}^{-1}\right)$. Note that the $J / D$-ratio used for the simulation is 1.25 , while the previously reported is 1.3 and the $g_{\text {rad }}$ used is 2.08 and not the one of the free electron. The MATLAB simulation script is available as supplementary information

been calculated by the fittings of the EXAFS data of Cpd-I reacted with peracid and trapped by rapid freeze-quenching, obtaining an average of $1.670 \AA$ for the $\mathrm{Fe}=\mathrm{O}$ bond and an average of $2.388 \AA$ for the Fe-S distance $[32,38]$. The shorter Fe-S distance found compared to that obtained for CPO was considered by the authors of the study as one of the potential factors responsible for the different reactivity of these two proteins. They proposed that the origin of this difference lies in the distinct strength of the hydrogen bonds between the axial thiolate and the surrounding structure, which are stronger in $\mathrm{CPO}$ than $\mathrm{P} 450$, thus reinforcing the $\mathrm{Fe}=\mathrm{O}$ bond due to lower electron donation into the ferryl $\pi^{*}$ orbitals $[38,39]$.

The Mössbauer spectrum of CYP119 (Table 1) is similar to the one of CPO [38]. Variable temperature-Mössbauer spectroscopy has been used to measure the electronic spin relaxation rates of CYP119 and CPO to obtain the zero-field splitting parameters. The spectra show a complex four-line pattern that collapses towards a quadrupole doublet with increasing temperature. Solving the spin Hamiltonian thanks to the results obtained with EPR spectroscopy, led to uniquely determine 
$J$ - and $D$-values, assuming an axial symmetry of zero-field splitting $(E / D=0)$. The results obtained are $D=40 \mathrm{~cm}^{-1}(58 \mathrm{~K})$ and $J=52 \mathrm{~cm}^{-1}(76 \mathrm{~K})$ for P450 Compound I [32], very close to the one obtained for CPO-I.

To measure CW EPR, CYP119 Cpd-I was obtained by reacting a highly purified CYP119 with m-chloroperbenzoic acid, and freezing the reaction at $3.5 \mathrm{~ms}$ [40]. Again, the CW-EPR spectrum was contaminated with the one from other species and was obtained by spectral subtraction after the study of their temperature and power dependence [38]. The EPR spectrum of the intermediate was also similar, regarding the overall characteristics, to the one of CPO-I, albeit with a smaller anisotropy (see Fig. 12b) in spite of conserving almost axial symmetry. The experimental data were simulated using an effective spin $S_{\text {eff }}=1 / 2$ with $g_{\text {eff }}=(1.86,1.96,2.00)$ values. Also, the spectrum was simulated using the two-spin spin Hamiltonian taking into account the ZFS parameters, $g_{\mathrm{Fe}(\mathrm{IV})}, g_{\text {radical, }}$ and an isotropic antiferromagnetic exchange interaction using the model presented in the previous paragraph (see Table 1 for the numerical values of the parameters). The best fits of the spectrum led to $J / D=1.3$ [38]. Similar studies were performed for Compound I of P450cam. The $g$-values obtained by fitting the effective representation with spin $S=1 / 2$ are indicative of antiferromagnetic coupling, and fit in the exchange-coupled representation yield $|J / D|=1.38$ close to that of CYP119 [40]. Although, in both studies, the authors seem unsure about using the $g_{\mathrm{Fe}(\mathrm{IV})}$-values of CPO, it is clear from the model presented above that $D$ and $g_{\mathrm{Fe}(\mathrm{IV})}$ 's are not independent quantities, but can be calculated from the crystal-field parameters $\Delta$ and $V$ (assumed to be $\approx 0$ ). Therefore, since the values found for $D$ are very similar to those of CPO, the values for $g_{\mathrm{Fe}(\mathrm{IV})}$ are expected to be similar, as well.

Several procedures have been tried to capture the Cpd-I from P450. Unfortunately, the various methods used have failed to trap the intermediate in sufficiently high concentrations to be studied with ENDOR [41].

In recent comparative studies between CPO and CYP119, efforts have been made to correlate the Fe-S distance with the isotropic exchange coupling constant $J$ and so to estimate the electron donation from the axial ligand. The authors consider that a bigger $J$ would indeed mean a shorter Fe-S distance and/or increased axial ligand spin density [32]. However, since the zero-field splitting is similar for both proteins, the difference in the ratio is only due to the exchange coupling interaction. However, it is surprising that the Fe-S distance would not affect the crystal-field parameters of Fe(IV).

We have also simulated the spectrum (see Fig. 12c) with the parameters reported in the figure caption.

\subsection{Catalase}

Catalases are oxidoreductases that act as antioxidants protecting the cell against oxidative stress, through reactions of reduction and disproportionation of $\mathrm{H}_{2} \mathrm{O}_{2}$ to water and dioxygen (see Fig. 13a). These enzymes have one of the highest turnover numbers ever found for an enzyme, this means that it is able to convert a very high number of peroxide molecules (in the order of millions) per second 
(a)

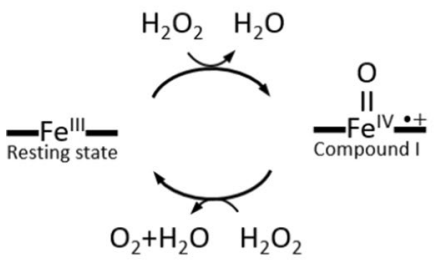

(c)

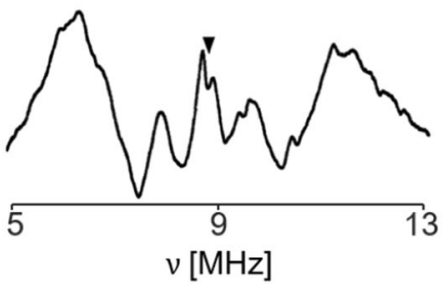

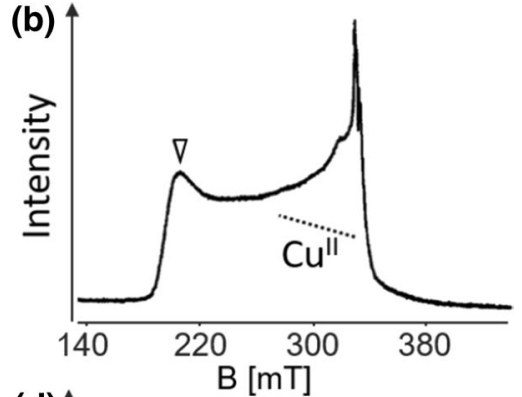

(d)

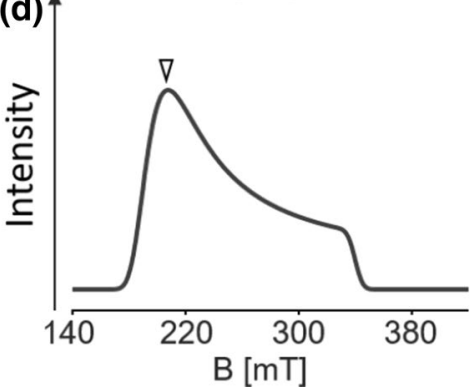

Fig. 13 a Reaction cycle of catalase. The resting state reacts with a molecule of hydrogen peroxide to form Compound I, that is converted back to the resting state through reaction with another peroxide molecule, with consequent generation of molecular oxygen. b Dispersion EPR spectrum of MCL-I produced by reaction with peracetic acid taken at $2 \mathrm{~K}$, adapted with permission from [47]. Copyright (1993) American Chemical Society. The spectrum extends from $g=3.44$ to approximately 2 . $\nabla$ Indicates $g_{\perp}$ $\approx 3.32$. At $g \approx 2, \mathrm{a} \mathrm{Cu}^{2+}$ impurity signal is visible. $\mathbf{c}$ ENDOR spectrum taken at $g=3.32$. $\nabla$ Indicates the free proton Larmor frequency. Adapted with permission from [47]. Copyright (1993) American Chemical Society. d Simulation performed with EasySpin using a Gaussian distribution of isotropic exchange parameters centered at $J=-8.5 \mathrm{~cm}^{-1}$ and FWHM of $6.8 \mathrm{~cm}^{-1}$. The rest of the parameters for the simulations are: $g_{\text {eff }}^{\mathrm{Fe}}=(2.15,2.15,1.99), D=17 \mathrm{~cm}^{-1}, E=0, J / D=-0.5$, corresponding to crystalfield parameters $\Delta=2680 \mathrm{~cm}^{-1}, V=0$ taking a spin-orbit coupling constant $\lambda=400 \mathrm{~cm}^{-1}$. $\nabla$ Indicates $g=3.32$. The MATLAB script used for the simulation is available as supplementary information

for each macromolecule [43]. This enzyme is found in nearly all organisms, aerobic and anaerobic, and it has been exploited in many applications. The industrial uses include food processing, textile, paper, pharmaceutical industry, and also in the field of bioremediation as one of the developing areas of its application [43]. Catalase can also act as a peroxidase, oxidizing alcohols, formate, or nitrite using hydrogen peroxide [44]. From the structural point of view, catalases are tetramers that contain four heme groups, with characteristics that vary according to the organism. The axial coordination is occupied by a phenolate ligand of a tyrosine side chain. In the distal pocket, a His residue is conserved and involved in assisting the breaking of the $\mathrm{O}-\mathrm{O}$ bond and proton transfer.

In the literature, a few atomic resolution structures of catalase Cpd-I are reported. The crystal structure of catalase Cpd-I from Proteus mirabilis (PMCI) was resolved at a resolution of $2.5 \AA$. The distances obtained were $1.76 \AA$ and 
$2.12 \AA$ for the $\mathrm{Fe}=\mathrm{O}$ and $\mathrm{Fe}-\mathrm{Tyr}$ bonds, respectively [45]. Also the Cpd-I from Penicillium vitale catalase (PVC) and Helicobacter pylori catalase (HPC) have been resolved by X-ray crystallography, but only PVC-I of the two contains a porphyrin $\pi$-cation radical specie with partial protonation of the distal histidine. This conclusion has been achieved by combining X-ray crystallography data and QM/ MM density functional theory calculations. The distances obtained at a resolution of $1.7 \AA$ are $1.72 \AA$ and $2.00 / 2.04 \AA$ (depending on the subunit) for the $\mathrm{Fe}=\mathrm{O}$ and Fe-Tyr bonds, respectively. The shorter distance is in accordance with the expected $\mathrm{Fe}=\mathrm{O}$ double bond in the proximal side [46].

Compound I of catalase is routinely generated when reacting the resting state of the enzyme with peracetic acid instead of the natural substrate hydrogen peroxide. The reason is that this "pseudo-substrate" induces a slower catalytic turnover and the obtained intermediate is relatively stable [48]. The EPR spectra of Cpd-I of different catalases have been reported showing different spectral behaviors. The EPR spectrum of Cpd-I of catalase from Micrococcus luteus (MCL-I) has been recorded under rapid passage conditions [47] (Fig. 13b). The system resembles the coupling model between the ferryl center and the porphyrin radical with a ferromagnetic exchange interaction $(J<0)$ and a ratio $|J| / D$ of 0.4 (see Fig. 13b). The resonance signal extends from 180 to $340 \mathrm{mT}$, with an axial $g$-tensor with $g_{\perp} \approx 3.32$ and $g_{/ /} \approx 2$ [47]. Preliminary ENDOR studies have been carried out on MCL-I, showing five sets of magnetically distinguishable protons, assigned to the porphyrin $\pi$-cation radical (see Fig. 13c). The signals are mirrored with respect to the proton Larmor frequency and are separated by hyperfine couplings $A^{\mathrm{H}}<\sim 6 \mathrm{MHz}$. None of the protons are deuterium exchangeable, suggesting that the ferryl moiety is not protonated. No ${ }^{14} \mathrm{~N}$ ENDOR has been observed, suggesting that the radical has an increased $A_{1 u}$ character, although the authors of the study could not exclude that this is caused by the experimental conditions [47]. This is in contrast to the results obtained for HRP-I, where maximum hyperfine couplings $A^{\mathrm{H}}<\sim 12-13 \mathrm{MHz}$, and ${ }^{14} \mathrm{~N}$ ENDOR studies have shown that HRP-I has predominantly $A_{2 u}$ symmetry.

Another EPR study of Cpd-I of the catalase from Proteus mirabilis and the one of bovine liver catalase (BLC-I) shows that both of them form the ferryl porphyrin $\pi$-cation radical to a different extent, when reacting with peracetic acid. The spectra have been recorded at 4.2 K. The PMC-I is relatively stable and shows a $\mathrm{pH}$ dependence generating two distinct forms, one with a ferromagnetic character (at $\mathrm{pH} \approx 5$ ) that resembles the signal of MCL-I, and one with a small exchange interaction (at $\mathrm{pH} \approx 7$ ) similar to the signal of HRP-I [49]. In a similar way, the EPR spectrum of BLC-I trapped at $40 \mathrm{~ms}$ after reaction with peracetic acid, showed a mixture of two forms that have been attributed to two different values for the magnetic exchange interactions. At longer reaction times, the signal of a tyrosine radical is predominant $[49,50]$.

Another Mössbauer study has been carried out on PMC-I at pH 5.8 [51], reproducing the experimental conditions used in the study of Schultz et al. for HRP [15]. The obtained results (Table 1) are in accordance with a low-spin configuration of $\mathrm{Fe}(\mathrm{IV})$. The Mössbauer spectra were successfully simulated using an antiferromagnetic exchange coupling constant $J=7.8 \mathrm{~K}\left(5.4 \mathrm{~cm}^{-1}\right)$ between 
the porphyrin $\pi$-cation radical and the ferryl iron and a positive zero-field splitting $D=25 \mathrm{~K}\left(17.2 \mathrm{~cm}^{-1}\right), J / D=0.31$ [51]. The authors reported that the $J$ - and $D$-values have been calculated independently using an isotropic $\boldsymbol{g}_{\mathrm{Fe}(\mathrm{IV})}=(2,2,2)$, which will reduce the correctness of the parameters obtained.

Our simulation of the experimental spectrum is shown in Fig. 13d using a distribution of isotropic and ferromagnetic $J$-values centered in $-8.5 \mathrm{~cm}^{-1}$.

\section{Conclusions}

Quantum-mechanical computations, such as DFT, are still challenging for high-spin iron systems. Hence, it is reassuring that EPR experimental data can be modeled with a simple model. The spin-coupling model revised in this article for Compound I is based on crystal-field theory and spin-spin interaction concepts. As illustrated with a set of examples of different enzymes, it is valid to interpret the experimental data collected to date with few parameters to be adjusted. From the derivation of the model, it is clear that the $g$-values and ZFS parameters of iron moiety in Compound I are not independent, but they are strictly correlated to the crystal-field parameters of the iron ion. This, although known in older literature analyses, in newer reports, it seems to be forgotten. Therefore, it is worth stressing that both g-values and ZFS have to be interpreted together to safely obtain the spin-spin interaction. However, interpreting the experimental spectra according to the model is only the first step. Still, many steps are needed to complete the interpretation of the magnetic parameters in structural and functional terms. Many questions are still open, such as: how does the location of Fe d-orbitals affect the exchange interaction?, how does the location of the radical affect reactivity?, and how does the protein matrix modulate the electronic states? These are very relevant points needed to fully understand the catalysis performed by the heme enzymes and to exploit this knowledge to develop mimics of the biocatalysts. As illustrated in this review, EPR experiments form a cornerstone in understanding the mechanistic pathways of the proteins.

Funding This paper is part of a project that has received funding from the European Union's Horizon 2020 research and innovation program under the Marie Skłodowska-Curie grant agreement No. 813209.

Availability of Data and Material This is an open-access article under the terms of the Creative Commons Attribution License (CC-BY 4.0 International), which permits use, distribution, and reproduction in any medium, provided that the original work is properly cited.

Code Availability The MATLAB scripts used for all simulations in this article are provided at the link: https://zenodo.org/record/4061955\#.X4QGhO1S_IV.

\section{Compliance with Ethical Standards}

Conflict of interest The authors declare no conflict of interest.

Open Access This article is licensed under a Creative Commons Attribution 4.0 International License, which permits use, sharing, adaptation, distribution and reproduction in any medium or format, as long as 
you give appropriate credit to the original author(s) and the source, provide a link to the Creative Commons licence, and indicate if changes were made. The images or other third party material in this article are included in the article's Creative Commons licence, unless indicated otherwise in a credit line to the material. If material is not included in the article's Creative Commons licence and your intended use is not permitted by statutory regulation or exceeds the permitted use, you will need to obtain permission directly from the copyright holder. To view a copy of this licence, visit http://creativecommons.org/licen ses/by/4.0/.

\section{References}

1. T.L. Poulos, Chem. Rev. 114, 3919 (2014)

2. E. Torres, M. Ayala, Biocatalysis Based on Heme Peroxidases (Springer, Berlin, 2010)

3. W.T. Oosterhuis, G. Lang, J. Chem. Phys. 58, 4757 (1973)

4. J. Krzystek, A. Schnegg, A. Aliabadi, K. Holldack, S.A. Stoian, A. Ozarowski, S.D. Hicks, M.M. Abu-Omar, K.E. Thomas, A. Ghosh, K.P. Caulfield, Z.J. Tonzetich, J. Telser, Inorg. Chem. 59, 1075 (2020)

5. J.S. Griffith, Proc. R. Soc. London. Ser. A. Math. Phys. Sci. 235, 23 (1956)

6. W.T. Oosterhuis, G. Lang, Phys. Rev. 178, 439 (1969)

7. P.J. Alonso, J.I. Martínez, I. García-Rubio, Coord. Chem. Rev. 251, 12 (2007)

8. M.P. Byrn, B.A. Katz, N.L. Keder, K.R. Levan, C.J. Magurany, K.M. Miller, J.W. Pritt, C.E. Strouse, J. Am. Chem. Soc. 105, 4916 (1983)

9. R. Rutter, L.P. Hager, H. Dhonau, M. Hendrich, P. Debrunner, M. Valentine, Biochemistry 23, 6809 (1984)

10. S.S. Eaton, G.R. Eaton, in Distance Measurements in Biological Systems by EPR, ed. by L.J. Berliner, G.R. Eaton, S.S. Eaton (Springer US, Boston, MA, 2000), pp. 29-154

11. R. Orbach, Proc. R. Soc. London. Ser. A. Math. Phys. Sci. 264, 458 (1961)

12. J.T. Colvin, R. Rutter, H.J. Stapleton, L.P. Hager, Biophys. J. 41, 105 (1983)

13. C.P. Scholes, R.A. Isaacson, G. Feher, Biochim. Biophys. Acta - Gen. Subj. 244, 206 (1971)

14. R.C. Herrick, H.J. Stapleton, J. Chem. Phys. 65, 4786 (1976)

15. C.E. Schulz, P.W. Devaney, H. Winkler, P.G. Debrunner, N. Doan, R. Chiang, R. Rutter, L.P. Hager, FEBS Lett. 103, 102 (1979)

16. S. McNicholas, E. Potterton, K.S. Wilson, M.E.M. Noble, Acta Crystallogr. Sect. D Biol. Crystallogr. 67, 386 (2011)

17. N.C. Veitch, Phytochemistry 65, 249 (2004)

18. N.C. Veitch, A.T. Smith, in Advances in Inorganic Chemistry, vol. 51 (2000), pp. 107-162.

19. G.I. Berglund, G.H. Carlsson, A.T. Smith, H. Szöke, A. Henriksen, J. Hajdu, Nature 417, 463 (2002)

20. H.P. Hersleth, U. Ryde, P. Rydberg, C.H. Görbitz, K.K. Andersson, J. Inorg. Biochem. 100, 460 (2006)

21. E. Raven, B. Dunford (eds.), Heme Peroxidases (The Royal Society of Chemistry, Cambride, UK, 2016)

22. M.T. Green, J.H. Dawson, H.B. Gray, Science 304, 1653 (2004)

23. R. Aasa, T. Vänngård, H.B. Dunford, BBA - Enzymol. 391, 259 (1975)

24. C.E. Schulz, R. Rutter, J.T. Sage, P.G. Debrunner, L.P. Hager, Biochemistry 23, 4743 (1984)

25. E. Bill, in Practical Approaches to Biological Inorganic Chemistry, ed. by R. Crichton, R. Louro (Elsevier, Oxford, UK, 2020), pp. 201-228

26. J.E. Roberts, B.M. Hoffman, R. Rutter, L.P. Hager, J. Am. Chem. Soc. 103, 7654 (1981)

27. J.E. Roberts, B.M. Hoffman, R. Rutter, L.P. Hager, J. Biol. Chem. 256, 2118 (1981)

28. S. Stoll, A. Schweiger, J. Magn. Reson. 178, 42 (2006)

29. X. Yi, M. Mroczko, K.M. Manoj, X. Wang, L.P. Hager, Proc. Natl. Acad. Sci. USA 96, 12412 (2002)

30. K.M. Manoj, L.P. Hager, Biochemistry 47, 2997 (2008)

31. E.G. Hrycay, S.M. Bandiera, in Advances in Experimental Medicine and Biology ed. by E.G. Hrycay, S.M. Bandiera (Springer International Publishing, Cham, 2015), pp. 1-61 
32. C.M. Krest, A. Silakov, J. Rittle, T.H. Yosca, E.L. Onderko, J.C. Calixto, M.T. Green, Nat. Chem. 7, 696 (2015)

33. K.L. Stone, R.K. Behan, M.T. Green, Proc. Natl. Acad. Sci. USA 102, 16563 (2005)

34. R. Rutter, L.P. Hager, J. Biol. Chem. 257, 7958 (1982)

35. S.H. Kim, R. Perera, L.P. Hager, J.H. Dawson, B.M. Hoffman, J. Am. Chem. Soc. 128, 5598 (2006)

36. I. Schlichting, J. Berendzen, K. Chu, A.M. Stock, S.A. Maves, D.E. Benson, R.M. Sweet, D. Ringe, G.A. Petsko, S.G. Sligar, Science 287, 1615 (2000)

37. C. Jung, S. De Vries, V. Schünemann, Arch. Biochem. Biophys. 507, 44 (2011)

38. J. Rittle, M.T. Green, Science 330, 933 (2010)

39. I.G. Denisov, S.G. Sligar, Nat. Chem. 7, 687 (2015)

40. T.H. Yosca, M.T. Green, Isr. J. Chem. 56, 834 (2016)

41. C.M. Krest, E.L. Onderko, T.H. Yosca, J.C. Calixto, R.F. Karp, J. Livada, J. Rittle, M.T. Green, J. Biol. Chem. 288, 17074 (2013)

42. E.G. Hrycay, S.M. Bandiera, in Monooxygenase, Peroxidase and Peroxygenase Properties and Mechanisms of Cytochrome P450, ed. by E.G. Hrycay, S.M. Bandiera (Springer International Publishing, Cham, 2015), pp. 1-61

43. J. Kaushal, S. Mehandia, G. Singh, A. Raina, S.K. Arya, Biocatal. Agric. Biotechnol. 16, 192 (2018)

44. G.R. Schonbaum, B. Chance, Enzymes 13, 363 (1976)

45. P. Andreoletti, A. Pernoud, G. Sainz, P. Gouet, H.M. Jouve, Acta Crystallogr. - Sect. D Biol. Crystallogr. 59, 2163 (2003)

46. M. Alfonso-Prieto, A. Borovik, X. Carpena, G. Murshudov, W. Melik-Adamyan, I. Fita, C. Rovira, P.C. Loewen, J. Am. Chem. Soc. 129, 4193 (2007)

47. M.J. Benecky, J.E. Frew, N. Scowen, P. Jones, B.M. Hoffman, Biochemistry 32, 11929 (1993)

48. P. Jones, D.N. Middlemiss, Biochem. J. 130, 411 (1972)

49. A. Ivancich, H.M. Jouve, B. Sartor, J. Gaillard, Biochemistry 36, 9356 (1997)

50. A. Ivancich, H.M. Jouve, J. Gaillard, J. Am. Chem. Soc. 118, 12852 (1996)

51. O. Horner, J.-M.M. Mouesca, P.L. Solari, M. Orio, J.-L.L. Oddou, P. Bonville, H.M. Jouve, J. Biol. Inorg. Chem. 12, 509 (2007)

Publisher's Note Springer Nature remains neutral with regard to jurisdictional claims in published maps and institutional affiliations. 